March 2000

IMF Staff Country Report No. 00/26

\title{
Mongolia: Statistical Annex
}

This Statistical Annex report on Mongolia was prepared by a staff team of the International Monetary Fund as background documentation for the periodic consultation with this member country. As such, the views expressed in this document are those of the staff team and do not necessarily reflect the views of the Government of Mongolia or the Executive Board of the IMF.

Copies of this report are available to the public from

International Monetary Fund - Publication Services

700 19th Street, N.W. - Washington, D.C. 20431

Telephone: (202) 623-7430 - Telefax: (202) 623-7201

Telex (RCA): 248331 IMF UR

E-mail: publications@imf.org

Internet: http://www.imf.org

Price: $\$ 15.00$ a copy

\section{International Monetary Fund Washington, D.C.}




\title{
INTERNATIONAL MONETARY FUND
}

\author{
MONGOLIA
}

\begin{abstract}
Statistical Annex
Prepared by Kevin Fletcher and Sanjay Kalra (APD), Catriona Purfield (FAD), and Sergei Dodzin (PDR)
\end{abstract}

Approved by Asia and Pacific Department

January 7,2000

Contents

Page

Tables

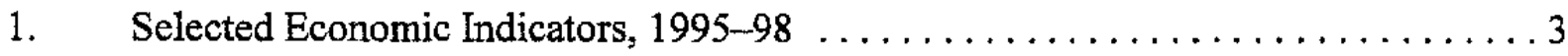

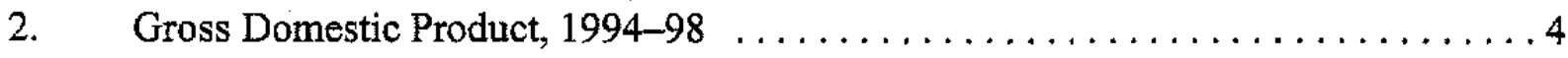

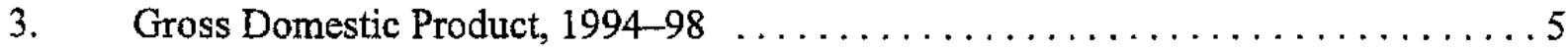

4. Output of Major Agricultural Products, $1994-98 \ldots \ldots \ldots \ldots \ldots \ldots \ldots \ldots$

5. Output of Basic Industrial and Mining Products, $1994-98 \ldots \ldots \ldots \ldots \ldots \ldots$

6. Composition of Gross Industrial Output, $1995-98 \ldots \ldots \ldots \ldots \ldots \ldots \ldots$

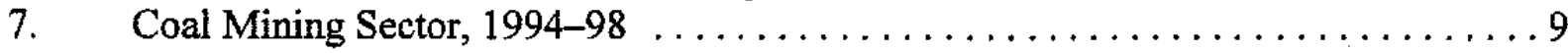

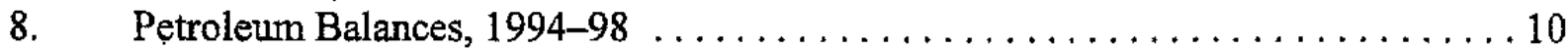

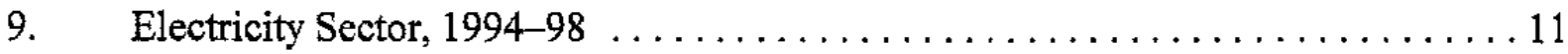

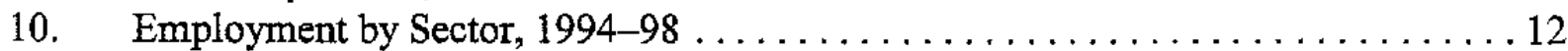

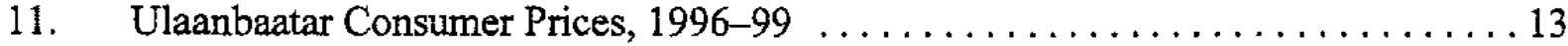

12. Ulaanbaatar Consumer Price Inflation, $1997-99 \ldots \ldots \ldots \ldots \ldots \ldots \ldots \ldots \ldots$

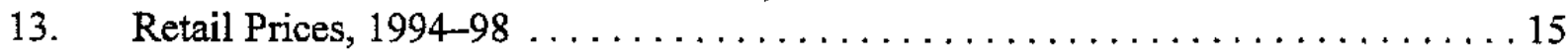

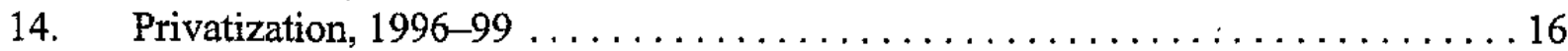

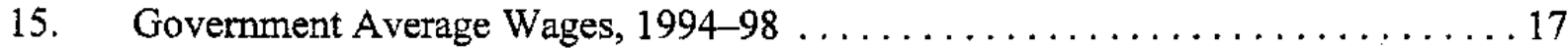

16. Summary Operations of the General Government, $1994-98 \ldots \ldots \ldots \ldots \ldots \ldots 18$

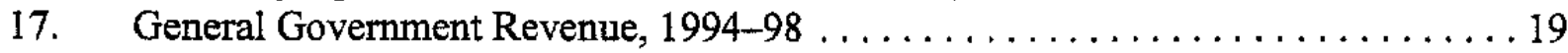

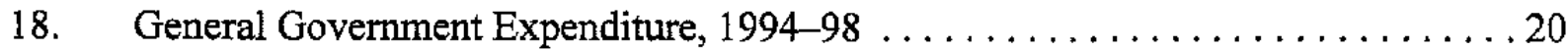

19. General Government Expenditure by Function, $1994-98 \ldots \ldots \ldots \ldots \ldots \ldots .21$

20. General Government Social Expenditure, $1994-98 \ldots \ldots \ldots \ldots \ldots \ldots \ldots 22$

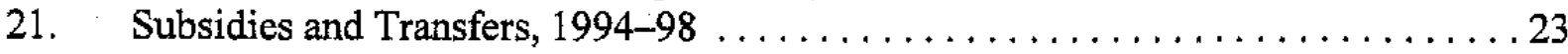

22. Social Security and Social Assistance Funds, $1994-98 \ldots \ldots \ldots \ldots \ldots \ldots 24$ 


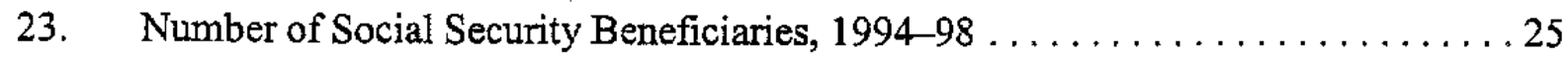

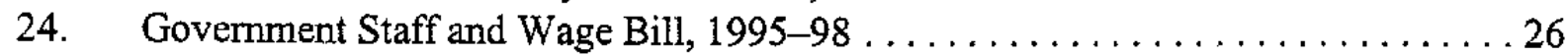

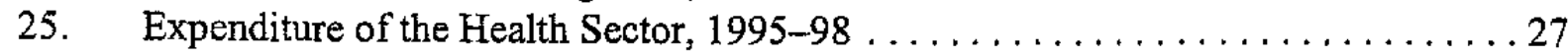

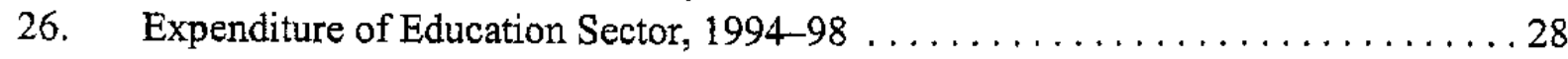

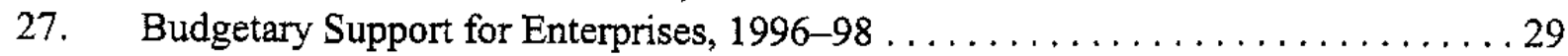

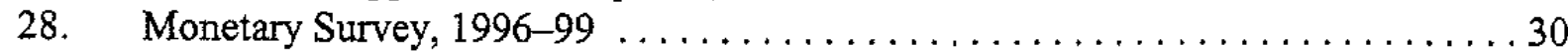

29. Balance Sheet of the Bank of Mongolia, 1996-99 ................... 31

30. Consolidated Balance Sheet of Commercial Banks, $1996-99$. . . . . . . . . . . 32

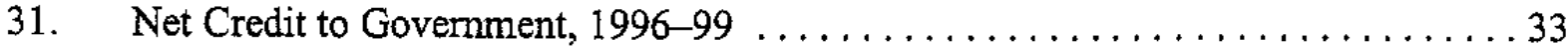

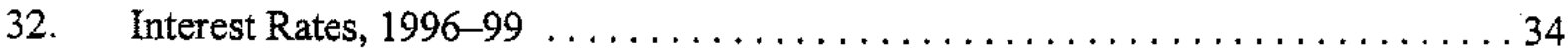

33. Selected Financial Indicators of Commercial Banks, $1998-99 \ldots \ldots \ldots \ldots \ldots 35$

34. Balance of Payments, 1994-98 . . . . . . . . . . . . . . . . . . . . . . 36

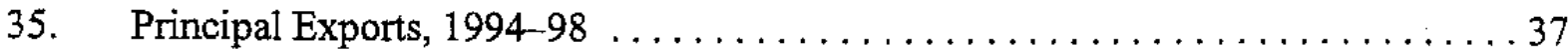

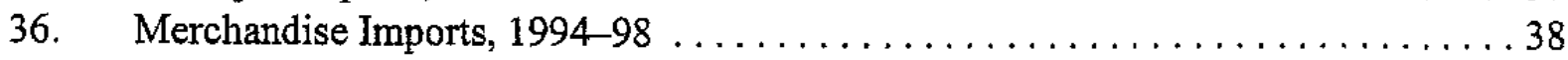

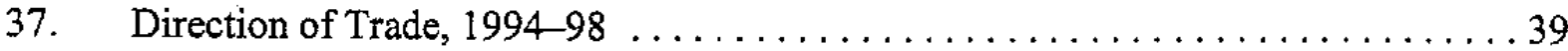

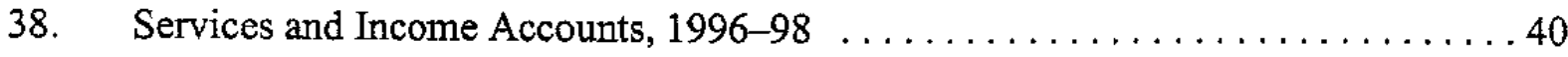

39. Medium- and Long-Term Loan Disbursements, $1994-98 \ldots \ldots \ldots \ldots \ldots \ldots . \ldots 4$

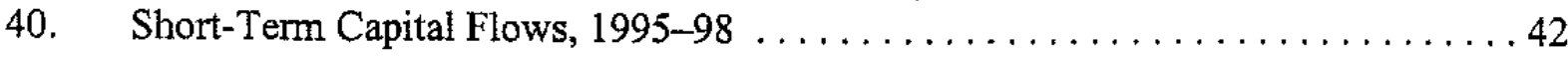

41. Official International Reserves of the Bank of Mongolia, $1995-98 \ldots \ldots \ldots \ldots 43$

42. Selected Indicators of Commercial Bank Foreign Exchange Operations, 1995-99 . . 44

43. External Debt and Debt Service in Convertible Currencies, 1994-98 . . . . . . . . 4 45

44. Monthly Exchange Rates, January 1996-September 1999 . . . . . . . . . . . . . 46

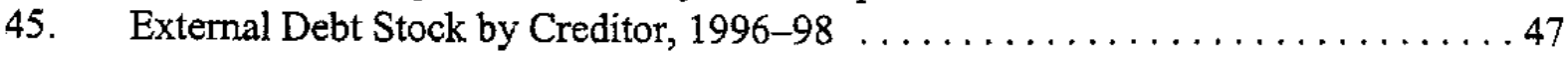

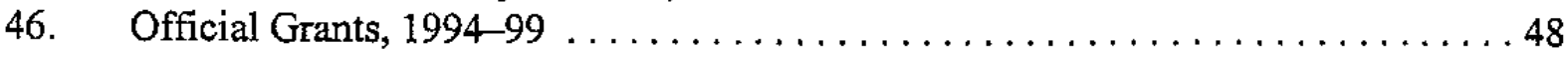

Appendix

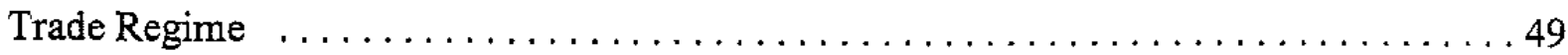


Table 1. Mongolia: Selected Economic Indicators, 1995-98

Nominal GDP (1998): $\$ 1026$ million

Population (1998): 2.36 million

Quota: SDR 51.1 million

1995

1996

1997

1998

\begin{tabular}{|c|c|c|c|c|}
\hline \multirow[b]{2}{*}{ Real GDP } & \multicolumn{3}{|c|}{ (Percent change) } & \multirow[b]{2}{*}{3.5} \\
\hline & 6.3 & 2,4 & 4.0 & \\
\hline Consumer prices (period average) & 56.9 & 46.9 & 36.6 & 9.4 \\
\hline \multirow[t]{2}{*}{ Consumer prices (end period) } & 53.3 & 44.6 & 20.5 & 6.0 \\
\hline & \multicolumn{3}{|c|}{ (In percent of GDP) } & \\
\hline General government revenue & 33.8 & 27.8 & 29.3 & 27.4 \\
\hline General government expenditure & 40.4 & 36,0 & 37.9 & 39.1 \\
\hline Current balance & 7.9 & 4.5 & 1.8 & -0.6 \\
\hline Overall balance & -6.7 & -8.2 & -8.6 & -11.7 \\
\hline \multirow[t]{2}{*}{ Domestic bank fimancing $1 /$} & -4.8 & 2.1 & -3.3 & 3.8 \\
\hline & \multicolumn{3}{|c|}{ (Percent change) } & \\
\hline Net foreign assets & 87.2 & 68,9 & 68.4 & -31.6 \\
\hline Net domestic assets & 12.4 & -1.4 & -6.3 & 56.4 \\
\hline Domestic credit & -21.7 & 133.6 & .5 .2 & 60.1 \\
\hline Credit to enterprises & 18.0 & 23.2 & 13.2 & 18.5 \\
\hline Broed money & 32.9 & 25.8 & 19.8 & 8.8 \\
\hline Reserve money & $\ldots$ & 36.2 & 26.2 & 13.5 \\
\hline Brond money velocity (GDP/BM) & 4.2 & 4.6 & 4.9 & 5.2 \\
\hline \multirow{2}{*}{ Anлual interest rate on central bank bills (percent) } & 71,3 & 63.3 & 40.9 & 22.5 \\
\hline & \multicolumn{3}{|c|}{ (In mitliors of U.S. dollars) } & \\
\hline \multirow{2}{*}{$\begin{array}{l}\text { Current account balance } 2 / \\
\text { (In percent of GDP) }\end{array}$} & .52 & -101 & 13 & -129 \\
\hline & -5.5 & -10.0 & 1.3 & $-12,6$ \\
\hline Trade balance & -3 & -87 & 30 & -120 \\
\hline (In percent of GDP) & -0.3 & -8.7 & 3.2 & -11.7 \\
\hline Exports, fob & 486 & 423 & 569 & 462 \\
\hline (Percent change) & 32.3 & -12.8 & 34.3 & -18.7 \\
\hline Imports, cif & 489 & SI1 & 538 & 582 \\
\hline (Percent change) & 32.0 & 4.5 & 5.4 & 8.2 \\
\hline Capital account balance & 19 & 41 & 53 & 117 \\
\hline (In percent of GDP) & 2.0 & 4.1 & 5.5 & 11.4 \\
\hline Gross official international reserves & 115 & 98 & 138 & 123 \\
\hline (In weeks of impons) & 12.2 & 10.0 & 13.3 & 11,0 \\
\hline Short-term debt (in percent of NIR of the BOM) $3 /$ & 66.7 & 69.1 & 36.3 & 38.8 \\
\hline External debt $4 /$ & 504 & 542 & 605 & 753 \\
\hline (In percent of GDP) & $\$ 2.8$ & 53.9 & 63.4 & 73.4 \\
\hline Debt service S/ & 64.2 & 56.6 & 41.0 & 39.6 \\
\hline (in percent of exports of goods \& services) & 12.1 & 11.8 & 6.3 & 7.3 \\
\hline \multicolumn{5}{|l|}{ Exchange tates and trade prices } \\
\hline Togrogs per U.S. dollar (end of period) & 474 & 694 & 813 & 915 \\
\hline Togrogs per U.S. dollar (period average) & 450 & 584 & 795 & 854 \\
\hline NEER, end-period (Dec 95=100) & 100.0 & 76.5 & 70.5 & 104.3 \\
\hline REER, end-period (Dec $95=100)$ & 100.0 & 106.8 & 108.9 & 153.8 \\
\hline Export prices (U.S. dollar, percent change) & 23.7 & -21.6 & 3.7 & -30.8 \\
\hline Copper price (U.S. dollar, percent charge) & 40.2 & -21.8 & -0.8 & -27.3 \\
\hline Import prices (U.S. dollar, percent change) & 9.1 & 2.6 & -7.5 & -9.7 \\
\hline Terms of trade (percent charge) & 13.4 & -23.6 & 12.2 & -23.3 \\
\hline Nominal GDP (billion togrogs) & 429 & 587 & 759 & 876 \\
\hline Nominal GDP (million U.S. dollars) & 954 & 1,006 & 955 & 1,026 \\
\hline
\end{tabular}

Sources: Mongolian authorities; and Fund staff estimates and projections.

1/ For 2000, higher-than-programmed costs of banking system reform and regularization of relations between the government and the BOM, up to an amount of Tog 5 billion, would be financed from the domestic banking system.

$2 /$ Excludes officiel transfers.

3/ Includes public sector amortization on medium- and long-term debt falling due during the period.

4/ Excludes Russia's unresolved 10.6 billion transferabie rouble claim.

5/ Excludes servicing of umresolved Russian medium- and long-term claims. 


$$
-4-
$$

Table 2. Mongolia: Gross Domestic Product, 1994-98

(At current prices)

\begin{tabular}{|c|c|c|c|c|c|}
\hline & 1994 & 1995 & 1996 & 1997 & 1998 \\
\hline & \multicolumn{5}{|c|}{ (In billions of togrogs) } \\
\hline Gross domestic product & 283.3 & 429.2 & 586.5 & 758.9 & 875.9 \\
\hline Industry & 93.3 & 119.3 & 120.6 & 183.0 & 211.5 \\
\hline Agriculture & 104.7 & 157.9 & 216.0 & 254.6 & 287.2 \\
\hline Construction & 6.0 & 11.5 & 22.2 & 25.4 & 30.2 \\
\hline Transportation & 12.9 & 14.6 & 27.6 & 39.8 & 46.7 \\
\hline Communications & 3.5 & 5.0 & 6.2 & 10.5 & 12.4 \\
\hline Trade & 33.3 & 72.7 & 107.5 & 140.0 & 169.3 \\
\hline Services & 29.7 & 48.2 & 86.4 & 105.6 & 118.7 \\
\hline Net factor income from abroad & -11.0 & -5.8 & -8.2 & -12.0 & -12.4 \\
\hline \multirow[t]{2}{*}{ Gross national product } & 272.2 & 423.4 & 578.4 & 746.9 & 863.5 \\
\hline & \multicolumn{5}{|c|}{ (In percent of GDP) } \\
\hline GDP & 100.0 & 100.0 & 100.0 & 100.0 & 100.0 \\
\hline Industry & 32.9 & 27.8 & 20.6 & 24.1 & 24.1 \\
\hline Agriculture & 36.9 & 36.8 & 36.8 & 33.5 & 32.8 \\
\hline Construction & 2.1 & 2.7 & 3.8 & 3.3 & 3.4 \\
\hline Transportation & 4.6 & 3.4 & 4.7 & 5.2 & 5.3 \\
\hline Communications & 1.2 & 1.2 & 1.1 & 1.4 & 1.4 \\
\hline Trade & 11.7 & 16.9 & 18.3 & 18.4 & 19.3 \\
\hline Services & 10.5 & 11.2 & 14.7 & 13.9 & 13.6 \\
\hline
\end{tabular}

Sources: Ministry of Finance; and Fund staff estimates. 
Table 3. Mongolia: Gross Domestic Product, 1994-98

(At 1993 constant prices)

\begin{tabular}{|c|c|c|c|c|c|}
\hline & 1994 & 1995 & 1996 & 1997 & 1998 \\
\hline & \multicolumn{5}{|c|}{ (In billions of togrogs) } \\
\hline Gross domestic product & 170.0 & 180.8 & 185.0 & 192.5 & 199.3 \\
\hline Industry & 56.6 & 61.3 & 60.0 & 62.7 & 64.7 \\
\hline Agriculture & 59.9 & 65.6 & 68.7 & 72.0 & 74.2 \\
\hline Construction & 3.0 & 3.3 & 3.5 & 3.3 & 3.5 \\
\hline Transportation & 5.4 & 5.3 & 5.7 & 5.9 & 6.1 \\
\hline Communication & 2.1 & 2.2 & 2.4 & 2.5 & 2.7 \\
\hline Trade & 26.5 & 26.6 & 27.2 & 28.3 & 29.6 \\
\hline Services & 16.5 & 16.6 & 17.5 & 17.7 & 18.6 \\
\hline Net factor income from abroad & -10.6 & -11.0 & -10.1 & -10.8 & -10.0 \\
\hline \multirow[t]{2}{*}{ Gross national product } & 159.4 & 169.8 & 175.0 & 181.7 & 189.3 \\
\hline & \multicolumn{5}{|c|}{ (Percent change) } \\
\hline GDP growth & 2.3 & 6.3 & 2.4 & 4.0 & 3.5 \\
\hline Industry & 1.7 & 8.3 & -2.0 & 4.4 & 3.2 \\
\hline Agriculture & 2.7 & 9.5 & 4.7 & 4.8 & 3.1 \\
\hline Construction & 10.5 & 10.6 & 5.0 & -4.9 & 4.4 \\
\hline Transportation & 0.1 & -2.1 & 7.3 & 4.2 & 3.5 \\
\hline Communication & -7.8 & 1.1 & 11.5 & 5.6 & 4.5 \\
\hline Trade & 0.0 & 0.1 & 2.5 & 4.1 & 4.5 \\
\hline Services & -0.2 & 0.5 & 5.6 & 1.3 & 4.8 \\
\hline Net factor income from abroad & -8.4 & 3.5 & -8.4 & 7.3 & -7.3 \\
\hline Gross national product & 3.1 & 6.5 & 3.1 & 3.8 & 4.2 \\
\hline
\end{tabular}

Sources: National Statistical Office, and the Ministry of Finance; and Fund staff estimates. 
Table 4. Mongolia: Output of Major Agricultural Products, 1994-98

\begin{tabular}{|c|c|c|c|c|c|}
\hline & 1994 & 1995 & 1996 & 1997 & 1998 \\
\hline & \multicolumn{5}{|c|}{ (In thousands of metric tons, unless otherwise specified) } \\
\hline Meat & 421 & 437 & 537 & 496 & 554 \\
\hline Milk (million tons) & 313 & 370 & 370 & 419 & 431 \\
\hline Butter (metric tons) & 800 & 1,200 & 653 & $\ldots$ & $\ldots$ \\
\hline Eggs (millions) & 3.6 & 3.5 & 4.9 & 6.1 & 8.5 \\
\hline Wool & 26 & 27 & 28 & 26 & 26 \\
\hline Cereals & 331 & 261 & 220 & 240 & 195 \\
\hline Potatoes & 54 & 52 & 46 & 55 & 65 \\
\hline \multirow[t]{2}{*}{ Other vegetables } & 23 & 27 & 24 & 34 & 46 \\
\hline & \multicolumn{5}{|c|}{ (In millions of heads) } \\
\hline Livestock & 26.8 & 28.6 & 29.3 & 31.3 & 32.9 \\
\hline Sheep & 13.8 & 13.7 & 13.5 & 14.2 & 14.7 \\
\hline Goats & 7.2 & 8.5 & 9.1 & 10.3 & 11.1 \\
\hline Cattle & 3.0 & 3.3 & 3.5 & 3.6 & 3.7 \\
\hline Horses & 2.4 & 2.7 & 2.8 & 2.9 & 3.1 \\
\hline \multirow[t]{2}{*}{ Camels } & 0.4 & 0.4 & 0.4 & 0.4 & 0.4 \\
\hline & \multicolumn{5}{|c|}{ (Percent change) } \\
\hline Meat & -5.4 & 3.8 & 22.9 & -7.6 & 11.7 \\
\hline Milk & 6.8 & 18.2 & 0.0 & 13.2 & 2.9 \\
\hline Butter & 12.4 & 50.0 & -45.6 & ,.. & $\ldots$ \\
\hline Eggs & -64.0 & -2.8 & 40.0 & 24.5 & 39.3 \\
\hline Wool & -7.1 & 3.8 & 3.7 & -7.1 & 0.0 \\
\hline Cereals & -30.9 & -21.1 & -15.7 & 9.1 & -18.8 \\
\hline Potatoes & -10.0 & -3.7 & -11.5 & 19.6 & 18.2 \\
\hline \multirow[t]{2}{*}{ Other vegetables } & 0.0 & 17.4 & -11.1 & 41.7 & 35.3 \\
\hline & \multicolumn{5}{|c|}{ (In percent of total heads) } \\
\hline \multicolumn{6}{|l|}{ Memorandum item: } \\
\hline Privately owned livestock & 91.5 & 92.6 & 93.5 & 94.4 & 95.3 \\
\hline
\end{tabular}

Sources: National Statistical Office, and the Ministry of Finance; and Fund staff estimates. 
Table 5. Mongolia: Output of Basic Industrial and Mining Products, 1994-98

(In thousands of metric tons, unless otherwise specified)

\begin{tabular}{|c|c|c|c|c|c|}
\hline & 1994 & 1995 & 1996 & 1997 & 1998 \\
\hline Electricity (million kilowatt hours) & 2,715 & 2,628 & 2,614 & 2,662 & 2,675 \\
\hline Coal & 5,158 & 5,019 & 5,110 & 4,924 & 5,057 \\
\hline Fluorspar & 383 & 527 & 565 & 567 & 612 \\
\hline Copper concentrate & 343 & 346 & 351 & 358 & 358 \\
\hline Gold concentrate (kilograms) & 1,789 & 4,504 & 6,976 & 8,451 & 9,531 \\
\hline Bricks (millions) & 34 & 22 & 25 & 16 & 19 \\
\hline Cement & 86 & 109 & 106 & 112 & 109 \\
\hline Lime & 66 & 51 & 55 & 58 & 56 \\
\hline Sawn timber (thousand of cubic meters) & 50 & 61 & 70 & 37 & 36 \\
\hline Scoured wool & 2 & 1 & 1 & 1 & 1 \\
\hline Felt (thousand meters) & 108 & 77 & 96 & 75 & 103 \\
\hline Woolen fabrics (thousand meters) & 77 & 71 & 45 & 8 & 5 \\
\hline Coats (thousands) & 1 & 0 & 0 & 2 & 0 \\
\hline Suits (thousands) & 3 & 1 & 1 & 1 & 2 \\
\hline Leather footwear (thousand pairs) & 407 & 246 & 87 & 42 & 33 \\
\hline Leather coats (thousands) & 35 & 13 & 5 & 0 & 0 \\
\hline Sheepskin coats (thousands) & 57 & 17 & 15 & 3 & 1 \\
\hline Meat (excluding pork) & 11 & 11 & 9 & 8 & 7 \\
\hline Sausages & 1,065 & 639 & 674 & 793 & 663 \\
\hline Flour & 127 & 159 & 92 & 64 & 66 \\
\hline Bakery goods & 34 & 37 & 30 & 20 & 19 \\
\hline Confectionery & 11 & 11 & 11 & 13 & 11 \\
\hline Milk and dairy products (liters) & 5 & 2 & 2 & 2 & 3 \\
\hline Toilet soap & 93 & 263 & 268 & 148 & $\cdots$ \\
\hline Household soap & 473 & 290 & 267 & 312 & 200 \\
\hline Carpets (thousands of square meters) & 682 & 596 & 666 & 644 & 588 \\
\hline Processed metal & 10 & 16 & 19 & 14 & $\cdots$ \\
\hline
\end{tabular}

Sources: National Statistical Office, and the Ministry of Finance; and Fund staff estimates. 
Table 6. Mongolia: Composition of Gross Industrial Output, 1995-98

(In percent)

\begin{tabular}{lrrrr}
\hline & 1995 & 1996 & 1997 & 1998 \\
& & & & \\
\hline & 100 & 100 & 100 & 100 \\
Gross output & 14.2 & 18.9 & 17.7 & 14.2 \\
Food & 0.9 & 0.7 & 0.4 & 0.4 \\
Leather and shoes & 10.5 & 9.4 & 8.1 & 8.0 \\
Textiles & 2.3 & 1.7 & 1.4 & 1.1 \\
Clothing & 13.3 & 13.0 & 17.2 & 19.4 \\
Energy 1/ & 4.3 & 5.2 & 5.3 & 6.2 \\
Coal & 44.4 & 38.8 & 38.2 & 37.9 \\
Mining of metal ores & 3.2 & 5.5 & 5.0 & 6.1 \\
Other mining and quarrying & 2.4 & 2.4 & 2.0 & 2.1 \\
Non-metalic products & 0.8 & 0.7 & 1.1 & 0.5 \\
Wood processing & 0.5 & 0.7 & 0.6 & 0.8 \\
Chemicals & 0.4 & 0.9 & 0.7 & 0.8 \\
Printing & 1.5 & 0.5 & 0.8 & 1.2 \\
Furniture & 1.3 & 1.6 & 1.5 & 1.3 \\
Other & & & & \\
\hline
\end{tabular}

Sources: National Statistical Office; and Fund staff estimates.

1/ Includes electric and thermal energy. 
Table 7. Mongolia: Coal Mining Sector, 1994-98

(In thousands of metric tons)

\begin{tabular}{|c|c|c|c|c|c|}
\hline & 1994 & 1995 & 1996 & 1997 & 1998 \\
\hline Production & 5,158 & 5,019 & 5,110 & 4,924 & 5,057 \\
\hline Imports & $\ldots$ & 211 & 23 & 100 & 38 \\
\hline Consumption & 5,167 & 5,204 & 5,139 & 5,035 & 4,986 \\
\hline Intermediate 1/ & 3,771 & 3,883 & 3,925 & 3,673 & 4,193 \\
\hline Final consumption & 1,396 & 1,321 & 1,214 & 1,362 & 793 \\
\hline Industry and construction & 592 & 651 & 555 & 856 & 465 \\
\hline Agriculture & 38 & 28 & 15 & 7 & 11 \\
\hline Communal housing and public services & 561 & 212 & 158 & 282 & 192 \\
\hline Other & 205 & 430 & 486 & 217 & 125 \\
\hline Exports & $\ldots$ & 1 & 1 & 0 & 3 \\
\hline \multicolumn{6}{|l|}{ Memorandum item: } \\
\hline Stock, end of year & 82 & 107 & 100 & 89 & 195 \\
\hline
\end{tabular}

Sources: National Statistical Office; and Fund staff estimates.

$1 /$ Consumption by thermal power stations. 
Table 8. Mongolia: Petroleum Balances, 1994-98

(In thousands of metric tons)

\begin{tabular}{|c|c|c|c|c|c|}
\hline & 1994 & 1995 & 1996 & 1997 & 1998 \\
\hline Opening Stocks & 185.3 & 141.1 & 96.9 & 63.7 & 65.1 \\
\hline Gasoline - octane 76 & 51.1 & 36.9 & 30.6 & 20.3 & 17.4 \\
\hline Gasoline - octane 93 & 2.3 & 0.5 & 0.9 & 1.1 & 1.7 \\
\hline Airplane fuel & 10.0 & 6.0 & 3.1 & 4.3 & 3.4 \\
\hline Diesel & 95.4 & 80.1 & 52.0 & 31.6 & 36.3 \\
\hline Other $1 /$ & 26.5 & 17.6 & 10.3 & 6.4 & 6.3 \\
\hline Imports & 264.1 & 228.6 & 282.4 & 185.5 & 195.9 \\
\hline Gasoline - octane 76 & 111.0 & 125.5 & 168.3 & 110.5 & 114.8 \\
\hline Gasoline - octane 93 & 3.6 & 6.0 & 11.5 & 17.0 & 21.4 \\
\hline Airplane fuel & 16.6 & 13.9 & 18.8 & 7.7 & 10.2 \\
\hline Diesel & 95.2 & 65.4 & 78.4 & 43.0 & 43.3 \\
\hline Other 1/ & 37.7 & 17.8 & 5.4 & 7.3 & 6.2 \\
\hline Domestic production 2/ & 214.0 & 254.6 & 66.7 & 68.9 & 17.3 \\
\hline Total supply & 663.4 & 624.3 & 446.0 & 318.1 & 278.3 \\
\hline Consumption 3/ & 332.3 & 320.1 & 379.1 & 251.9 & 224.1 \\
\hline Losses & 190.0 & 207.3 & 3.2 & 1.1 & 0.9 \\
\hline Closing stocks & 141.1 & 96.9 & 63.7 & 65.1 & 53.3 \\
\hline Gasoline - octane 76 & 36.9 & 30.6 & 20.3 & 17.4 & 18.6 \\
\hline Gasoline - octane 93 & 0.5 & 0.9 & 1.1 & 1.7 & 1.8 \\
\hline Airplane fuel & 6.0 & 3.1 & 4.3 & 3.4 & 2.9 \\
\hline Diesel & 80.1 & 52.0 & 31.6 & 36.3 & 24.7 \\
\hline Other $1 /$ & 17.6 & 10.3 & 6.4 & 6.3 & 5.3 \\
\hline
\end{tabular}

Sources: Neft Import Concern; and Fund staff estimates.

1/ Fuel for electricity generation and lubricants.

2/ Purchased domestically.

3/ Sales of Neft Import Concern. 
Table 9. Mongolia: Electricity Sector, 1994-98

\begin{tabular}{|c|c|c|c|c|c|}
\hline & 1994 & 1995 & 1996 & 1997 & 1998 \\
\hline & \multicolumn{5}{|c|}{ (In millions of kilowatt hours) } \\
\hline Supply & 2,930 & 3,009 & 2,997 & 3,006 & 3,042 \\
\hline Domestic & 2,715 & 2,628 & 2,614 & 2,662 & 2,675 \\
\hline Imports & 215 & 381 & 383 & 344 & 367 \\
\hline Utilization, total & 2,930 & 3,009 & 2,997 & 3,006 & 3,042 \\
\hline Industry and construction & 1,189 & 1,105 & 1,226 & 1,261 & 1,210 \\
\hline Transport and communications & 59 & 69 & 62 & 76 & 63 \\
\hline Agriculture & 49 & 59 & 60 & 32 & 11 \\
\hline Commercial housing and public services & 430 & 518 & 380 & 400 & 426 \\
\hline Other sectors & 134 & 158 & 208 & 113 & 219 \\
\hline Loss in transmission and distribution & 472 & 502 & 482 & 493 & 465 \\
\hline Power stations' own use & 597 & 598 & 579 & 589 & 588 \\
\hline \multirow[t]{2}{*}{ Exports } & $\ldots$ & $\ldots$ & $\ldots$ & 42 & 60 \\
\hline & \multicolumn{5}{|c|}{ (Togrogs per kilowatt hour, annual average) } \\
\hline \multicolumn{6}{|l|}{ Tariff } \\
\hline Industry & 13.0 & 17.5 & 34.0 & 37.0 & 37.0 \\
\hline Agriculture & 13.0 & 17.5 & 34.0 & 37.0 & 37.0 \\
\hline \multirow[t]{2}{*}{ Househoids } & 10.0 & 16.6 & 26.0 & 32.0 & 32.0 \\
\hline & \multicolumn{5}{|c|}{ (Percent change) } \\
\hline Supply and utilization & 5.4 & 2.7 & -0.4 & 0.3 & 1.2 \\
\hline Domestic & 5.2 & -3.2 & -0.5 & 1.8 & 0.5 \\
\hline Imports & 9.1 & 77.2 & 0.5 & -10.2 & 6.7 \\
\hline Industry and construction & 1.0 & -7.1 & 11.0 & 2.9 & -4.0 \\
\hline Transport and communications & -33.0 & 16.9 & -10.1 & 22.6 & -17.1 \\
\hline Agriculture & 4.2 & 20.4 & 1.7 & -46.7 & -65.6 \\
\hline Commercial housing and public services & 38.7 & 20.5 & -26.6 & 5.3 & 6.5 \\
\hline Other sectors & -67.1 & 17.9 & 31.6 & -45.7 & 93.8 \\
\hline \multicolumn{6}{|l|}{ Loss in transmission and power stations' } \\
\hline own use in total supply (in percent) & 36.5 & 36.6 & 35.4 & 36.0 & 34.6 \\
\hline
\end{tabular}

Sources: National Statistical Office; and Fund staff estimates. 
Table 10. Mongolia: Employment by Sector, 1994-98

(Number of employees, in thousands at end of year)

\begin{tabular}{|c|c|c|c|c|c|}
\hline & 1994 & 1995 & 1996 & 1997 & 1998 \\
\hline Total employment & 786.5 & 794.7 & 791.8 & 788.3 & 809.5 \\
\hline Agriculture and forestry & 339.6 & 354.2 & 358.1 & 374.5 & 394.1 \\
\hline Industry & 99.9 & 108.1 & 104.6 & 100.4 & 97.9 \\
\hline Transport and communications & 31.5 & 31.6 & 31.6 & 30.3 & 33.4 \\
\hline Construction & 27.3 & 29.5 & 29.7 & 27.4 & 27.5 \\
\hline Education 1/ & 51.9 & 48.5 & 46.0 & 43.0 & 42.5 \\
\hline Health & 45.9 & 38.1 & 37.1 & 35.4 & 35.6 \\
\hline Other & 163.7 & 157.6 & 162.5 & 154.1 & 161.6 \\
\hline Foreign employees & 26.7 & 27.1 & 22.2 & 23.2 & 16.9 \\
\hline \multicolumn{6}{|l|}{ Memorandum items: } \\
\hline Labor force & 861.4 & 839.8 & 847.2 & 852.0 & 859.3 \\
\hline Unemployed & 74.9 & 45.1 & 55.4 & 63.7 & 49.8 \\
\hline
\end{tabular}

Sources: National Statistical Office; and Fund staff estimates.

1/ Includes science, research, and development, 
Table 11. Mongolia: Ulaanbaatar Consumer Prices, 1996-99 1/

(December $1995=100)$

\begin{tabular}{|c|c|c|c|c|}
\hline & 1996 & 1997 & 1998 & $\begin{array}{c}1999 \\
\text { Jan.-Sept. 2f }\end{array}$ \\
\hline & \multicolumn{4}{|c|}{ (Period average) } \\
\hline Overali index & 123.6 & 168.8 & 184.6 & 198.3 \\
\hline Food and beverages & 125.0 & 156.9 & 160.2 & 168.6 \\
\hline Clothing & 114.8 & 160.6 & 197.0 & 214.0 \\
\hline Rent and utilities & 124.3 & 215.8 & 262.5 & 293.4 \\
\hline Household goods & 115.2 & 160.6 & 162.4 & 159.4 \\
\hline Medical care & 121.2 & 178.3 & 188.0 & 200.2 \\
\hline Transport and communications & 127.5 & 195.5 & 224.8 & 249.3 \\
\hline Education and recreation & 122.7 & 192.5 & 243.6 & 278.6 \\
\hline \multirow[t]{2}{*}{ Other goods and services } & 124.9 & 172.9 & 199.6 & 216.5 \\
\hline & \multicolumn{4}{|c|}{ (End of period) } \\
\hline Overall index & 144.6 & 174.2 & 184.7 & 194.4 \\
\hline Food and beverages & $\mathrm{L} 33.7$ & 148.8 & 150.1 & 156.5 \\
\hline Clothing & 146.0 & 193.4 & 213.8 & 216.1 \\
\hline Rent and utilities & 172.7 & 260.1 & 284.2 & 298.2 \\
\hline Household goods & 142.3 & 170.6 & 159.0 & 163.1 \\
\hline Medical care & 147.7 & 184.1 & 188.9 & 222.5 \\
\hline Transport and conmunications & 179.5 & 198.8 & 236.0 & 256.9 \\
\hline Education and recreation & 156.6 & 224.1 & 268.4 & 308.6 \\
\hline \multirow[t]{2}{*}{ Other goods and services } & 151.8 & 182.5 & 211.8 & 222.3 \\
\hline & \multicolumn{4}{|c|}{ (Percent change) } \\
\hline \multicolumn{5}{|l|}{ Memorandum items: } \\
\hline Annual average & $\ldots$ & 36.6 & 9.4 & 10.0 \\
\hline End of period & 44.6 & 20.5 & 6.0 & 7.1 \\
\hline
\end{tabular}

Sources: National Statistical Office; and Fund staff estimates.

1/ The number of commodities comprising the consumer basket was increased from 123 to 205 and new weights were adopted in January 1996.

2/ Percent change for 1999 is the change from 1998 to the first 9 months of 1999 at an annualized rate. 
Table 12. Mongolia: Ulaanbataar Consumer Price Inflation, 1997-99

(12-month percentage change)

\begin{tabular}{|c|c|c|c|c|c|c|c|c|c|c|c|c|c|c|c|c|c|c|c|c|c|c|c|c|c|}
\hline & \multirow[b]{2}{*}{ Weight } & \multirow{2}{*}{$\begin{array}{r}1997 \\
-12\end{array}$} & \multicolumn{12}{|c|}{1998} & \multicolumn{11}{|c|}{1999} \\
\hline & & & 1 & 2 & 3 & 4 & 5 & 6 & 7 & 8 & 9 & 10 & 11 & $\sqrt{12}$ & 1 & 2 & $\overline{3}$ & $\overline{4}$ & 5 & 6 & 7 & 8 & 9 & 10 & $\overline{11}$ \\
\hline Overall index & 1.00 & 20.5 & 21.0 & 17.1 & 19.1 & 15.5 & 10.3 & 2.1 & 2.5 & 7.4 & 5.7 & 5.3 & 4.9 & 6.0 & 3.9 & 7.1 & 4.4 & 4.2 & 5.6 & 8.6 & 8.7 & 9.6 & 9.8 & 8.9 & 10.4 \\
\hline Foodstuffs & 0.59 & 11.3 & 11.9 & 6.0 & 14.3 & 9.8 & 3.2 & -5.8 & -5.7 & 0.9 & -3.2 & -2.0 & -1.3 & 0.9 & -2.2 & 3.4 & -1.9 & -2.3 & 0.4 & 5.7 & 4.8 & 5.8 & 9.0 & 6.4 & 8.1 \\
\hline Flour and flour goods & 0.17 & 4.2 & -1.7 & -4.4 & -4.1 & -3.8 & -4.2 & -6.9 & -7.7 & -5.1 & -3.2 & -1.4 & -2.1 & -1.8 & -0.9 & -0.7 & 0.0 & -0.4 & 0.2 & 0.7 & 1.4 & 5.9 & 3.1 & 1.0 & 3.9 \\
\hline Meat and meat products & 0.18 & 8.9 & 18.7 & 0.7 & 39.0 & 28.4 & 9.1 & -8.6 & -10.0 & 0.7 & -8.6 & -8.7 & -1.8 & 3.8 & -9.4 & 10.3 & -13.1 & -14.3 & -4.7 & 8.9 & 6.9 & 0.9 & 8.3 & 6.5 & 5.8 \\
\hline Millk and dairy products & 0.05 & 35.9 & 34.8 & 27.8 & 17.8 & 5.5 & 0.9 & 1.0 & 0.4 & -0.4 & -4.4 & -1.8 & 3.2 & 4.2 & 3.7 & 2.1 & 1.7 & 9.0 & 9.7 & 4.3 & 1.4 & 9.3 & 13.0 & 7.0 & 4.1 \\
\hline Sugar, candy, tea, and fruits & 0.03 & 16.9 & 13.5 & 14.6 & 7.4 & 2.0 & 0.5 & 0.8 & -1.7 & -3.9 & -3.7 & -5.4 & -6.8 & -5.8 & -2.2 & -1.0 & 1.0 & 2.5 & 3.3 & 4.5 & 7.4 & 11.7 & 10.0 & 11.4 & 11.3 \\
\hline Potatoes and vegetables & 0.05 & -0.2 & 3.6 & 11.3 & 5.7 & -9.5 & -9.1 & -22.9 & -7.1 & 20.6 & 1.4 & -11.6 & -13.3 & -10.1 & -12.8 & -9.4 & 3.6 & 7.7 & -5.9 & -1.1 & -1.7 & 6.7 & 29.4 & 37.2 & 51.4 \\
\hline Other foodstuffis & 0.05 & 17.1 & 18.7 & 19.9 & 15.3 & 12.2 & 10.7 & 10.4 & 0.5 & 3.8 & 3.7 & 15.4 & 13.1 & 15.9 & 13.9 & 14.3 & 19.7 & 17.9 & 17.6 & 17.3 & 16.9 & 15.5 & 17.7 & 5.7 & 6.1 \\
\hline Alcohol and tobacco & 0.05 & 23.4 & 25.7 & 19.2 & 12.2 & 13.8 & 16.1 & 15.0 & 14.2 & 9.3 & 8.1 & 7.7 & -4.9 & -4.3 & 0.7 & 2.7 & 4.6 & 4.6 & 4.6 & 4.9 & 5.1 & 6.2 & 6.2 & 6.1 & 6.5 \\
\hline Clothes and shoes & 0.10 & 32.5 & 33.7 & 33.1 & 28.7 & 26.2 & 22.4 & 18.7 & 20.5 & 22.6 & 24.2 & 19.5 & 17.8 & 10.5 & 10.4 & 11.5 & 11.2 & 11.3 & 12.3 & 12.9 & 13.1 & 10.7 & 7.3 & 6.3 & 5.9 \\
\hline Men's clothing & 0.02 & 28.1 & 25.9 & 29.2 & 23.1 & 22.4 & 17.6 & 15.1 & 14.1 & 23.3 & 17.5 & 14.5 & 11.1 & 9.4 & 10.5 & 10.9 & 5.5 & 4.9 & 4.9 & 5.3 & 5.2 & -1.8 & -2.4 & -2.9 & .0 .9 \\
\hline Wornen's clothing & 0.03 & 42.5 & 42.8 & 41.7 & 36.5 & 32.3 & 28.7 & 24.8 & 22.2 & 19.9 & 19.5 & 18.0 & 12.8 & 5.1 & 4.1 & 8.4 & 7.2 & 7.6 & 8.5 & 8.8 & 8.5 & 8.9 & 7.5 & 2.8 & 6.1 \\
\hline Children's clothing & 0.01 & 27.3 & 24.3 & 29.5 & 21.2 & 18.7 & 13.8 & 7.2 & 8.1 & 8.3 & 19.7 & 10.9 & 7.0 & 5.7 & 2.1 & -0.4 & -1.1 & -1.1 & 1.2 & 3.7 & 4.2 & 4.4 & -1.2 & -1.2 & -0.9 \\
\hline Footwear & 0.04 & 29.8 & 34.3 & 30.3 & 28.0 & 26.8 & 22.9 & 19.8 & 26.4 & 30.2 & 33.3 & 26.7 & 29.1 & 17.5 & 18.7 & 18.4 & 20.5 & 20.4 & 21.7 & 21.4 & 22.3 & 19.1 & 13.0 & 14.3 & 10.2 \\
\hline Cloth & 0.00 & 16.2 & 14.9 & 22.8 & 18.0 & 9.9 & 11.3 & 5.3 & 4.1 & 3.5 & 6.3 & -0.5 & -1.8 & -1.9 & -4.9 & -6.6 & -4.2 & -3.6 & -4.1 & 1.6 & -0.1 & -2.5 & -2.7 & -3.3 & -3.9 \\
\hline Housing heating, and electricity & 0.10 & 50.6 & 47.1 & 47.8 & 44.8 & 43.4 & 34.6 & 11.5 & 12.5 & 12.3 & 11.6 & 7.7 & 6.1 & 9.2 & 8.9 & 13.6 & 13.7 & 15.2 & 14.5 & 15.0 & 14.9 & 14.8 & 13.5 & 10.6 & 9.3 \\
\hline Household goods & 0.04 & 19.9 & 18.1 & 14.1 & 5.0 & 4.3 & 5.9 & 3.0 & -1.6 & -2.7 & -2.0 & -8.3 & -10.2 & -6.8 & -7.0 & -6.9 & -4.6 & -3.5 & -4.4 & -2.8 & 0.3 & 3.6 & 3.1 & 2.6 & 4.9 \\
\hline Medical care & 0.02 & 24.7 & 26.7 & 24.5 & 0.1 & 0.1 & 2.2 & 2.7 & 2.7 & 3.2 & 2.6 & 2.6 & 2.6 & 2.6 & 1.1 & 1.1 & 1.3 & 1.0 & 1.0 & 0.6 & 18.2 & 17.6 & 17.8 & 17.8 & 17.8 \\
\hline Tratsport and communication & 0.08 & 10.7 & 16.7 & 21.4 & 15.7 & 10.6 & 10.7 & 9.8 & 10.1 & 10.1 & 18.5 & 19.5 & 18.8 & 18.7 & 14.5 & 9.3 & 12.5 & 12.6 & 12.5 & 12.8 & 12.7 & 19.0 & 9.2 & 18.3 & 27.4 \\
\hline Education and recreation & 0.04 & 43.1 & 41.9 & 32.2 & 25.9 & 26.9 & 27.0 & 29.8 & 29.8 & 35.8 & 21.6 & 18.8 & 17.4 & 19.7 & 18.2 & 18.8 & 19.4 & 18.6 & 18.6 & 18.2 & 19.3 & 14.5 & 16.7 & 16.7 & 17.1 \\
\hline Other goods and services & 0.03 & 20.3 & 19.7 & 16.2 & 12.6 & 12.8 & 12.6 & 11.6 & 12.8 & 18.3 & 17.3 & 20.1 & 15.5 & 16.0 & 15.7 & 15.2 & 11.7 & 13.3 & 12.3 & 7.3 & 7.3 & 8.8 & 5.6 & 5.9 & 6.2 \\
\hline
\end{tabular}

Sources: National Statistical Office; and Fund staff estimates. 
Table 13. Mongolia: Retail Prices, 1994-98

(End of period; in togrogs per kilogram, except where stated)

\begin{tabular}{lccccc}
\hline & 1994 & 1995 & 1996 & 1997 & 1998 \\
\hline Mutton & 320 & 550 & 550 & 595 & 620 \\
Beef & 360 & 550 & 650 & 650 & 700 \\
Flour & 118 & 196 & 325 & 365 & 325 \\
Bread & 90 & 140 & 240 & 230 & 240 \\
Sugar & 340 & 450 & 550 & 555 & 500 \\
Rice & 250 & 360 & 390 & 360 & 390 \\
Vegetable oil (liter) & 850 & 1,000 & 1,500 & 1,400 & 1,570 \\
Tea bricks (2 kilograms) & 2,600 & 1,900 & 1,800 & 2,465 & 2,260 \\
Tobacco & 600 & 550 & 1,200 & 1,400 & 1,290 \\
Gasoline A-93 (liter) & 164 & 164 & 235 & 235 & 265 \\
Gasoline A-76 (liter) & 126 & 126 & 200 & 206 & 226 \\
Diesel (liter) & 151 & 151 & 237 & 222 & 249 \\
\hline
\end{tabular}

Sources: Ministry of Finance, National Statistical Office, and Neft Import Concern; and Fund staff estimates. 
Table 14. Mongolia: Privatization, 1996-99

\begin{tabular}{|c|c|c|}
\hline & $\begin{array}{r}\text { No. of } \\
\text { Enterprises } \\
\text { Privatized }\end{array}$ & $\begin{array}{r}\text { Privatization } \\
\text { Revenue } \\
\text { (million togrogs) }\end{array}$ \\
\hline \multicolumn{3}{|l|}{1996} \\
\hline Total privatization & 13 & 1,500 \\
\hline Large enterprises & 0 & 0 \\
\hline Construction sector & $\cdots$ & $\cdots$ \\
\hline Mineral sector & $\cdots$ & $\cdots$ \\
\hline Agriculture sector & $\ldots$ & $\ldots$ \\
\hline Other & $\cdots$ & $\ldots$ \\
\hline Small enterprises and assets & 13 & 1,500 \\
\hline \multicolumn{3}{|l|}{1997} \\
\hline Total privatization & 236 & 16,650 \\
\hline Large enterprises & 3 & 1,138 \\
\hline Construction sector & 0 & 0 \\
\hline Mineral sector & 0 & 0 \\
\hline Agriculture sector & 1 & 78 \\
\hline Other & 2 & 1,080 \\
\hline Small enterprises and assets & 233 & 15,512 \\
\hline \multicolumn{3}{|l|}{1998} \\
\hline Total privatization & 206 & 15,116 \\
\hline Large enterprises & 52 & 7,462 \\
\hline Construction sector & 14 & 1,251 \\
\hline Mineral sector & 4 & 1,334 \\
\hline Agriculture sector & 16 & 2,224 \\
\hline Other & 18 & 2,653 \\
\hline Small enterprises and assets & 154 & 7,654 \\
\hline \multicolumn{3}{|l|}{1999 (January-October) } \\
\hline Total privatization & 87 & 8,939 \\
\hline Large enterprises & 24 & 7,144 \\
\hline Construction sector & 2 & 199 \\
\hline Mineral sector & 2 & 918 \\
\hline Agriculture sector & 9 & 2,749 \\
\hline Other & 11 & 3,278 \\
\hline Small enterprises and assets & 63 & 1,796 \\
\hline
\end{tabular}

Sources: State Property Commission; and Fund staff estimates. 
Table 15: Mongolia: Government Average Wages, 1994-98

\begin{tabular}{lrrrrr}
\hline & 1994 & 1995 & 1996 & 1997 & 1998 \\
\hline Employment (thousands) & 152.7 & 151.5 & 141.9 & 136.8 & 135.2 \\
Wage bill (billions of togrogs) & 16.1 & 26.8 & 36.7 & 44.8 & 56.0 \\
Per capita wage (thousands of togrogs) & 105.5 & 176.6 & 258.7 & 327.5 & 414.2 \\
Nominal wage index (1996=100) & 40.8 & 68.3 & 100.0 & 126.6 & 160.1 \\
$\quad$ Percent change & 93.5 & 67.4 & 46.5 & 26.6 & 26.5 \\
CPI index (period average, 1996=100) & 43.4 & 68.1 & 100.0 & 136.6 & 149.4 \\
$\quad$ Percent change & 87.6 & 56.9 & 46.9 & 36.6 & 9.4 \\
Real wage index (1996=100) & 94.0 & 100.3 & 100.0 & 92.6 & 107.1 \\
$\quad$ Percent change & 3.1 & 6.7 & -0.3 & -7.4 & 15.6 \\
Exchange rate (Togrogs/US\$) & 410 & 450 & 584 & 795 & 854 \\
Monthly per capita wage (US\$) & 21.4 & 32.7 & 36.9 & 34.3 & 40.4 \\
\hline
\end{tabular}

Sources: Ministry of Finance and National Statistical Office; and Fund staff estimates. 
Table 16. Mongolia: Summary Operations of the General Government, 1994-98

\begin{tabular}{|c|c|c|c|c|c|}
\hline & 1994 & 1995 & 1996 & 1997 & 1998 \\
\hline & \multicolumn{5}{|c|}{ (In billions of togrogs) } \\
\hline Total revenue & 86.1 & 144.9 & 162.9 & 222.5 & 240.0 \\
\hline Current revenue & 82.2 & 135.7 & 155.9 & 206.0 & 216.8 \\
\hline Tax revenue and social security contributions & 67.5 & 105.5 & 120.9 & 164.0 & 154.0 \\
\hline Income taxes & 32.0 & 48.5 & 45.6 & 62.6 & 41.5 \\
\hline Taxes on goods and services & 19.5 & 28.1 & 37.2 & 63.3 & 76.9 \\
\hline Customs duties & 7.5 & 9.6 & 13.9 & 8.8 & 1.5 \\
\hline Social security contributions & 6.3 & 15.8 & 18.5 & 21.2 & 26.1 \\
\hline Other taxes & 2.1 & 3.5 & 5.7 & 8.0 & 8.0 \\
\hline Nontax revenue & 14.7 & 30.2 & 35.0 & 42.0 & 62.8 \\
\hline Capital revenue and privatization proceeds & 0.7 & 3.9 & 2.6 & 10.5 & 14.6 \\
\hline Grants & 3.3 & 5.1 & 4.4 & 6.0 & 8.6 \\
\hline Total expenditure and net lending & 122.9 & 173.3 & 211.3 & 287.6 & 342.1 \\
\hline Current expenditure & 72.2 & 101.5 & 129.8 & 192.6 & 222.4 \\
\hline Wages and salaries & 16.1 & 26.8 & 36.7 & 44.8 & 56.0 \\
\hline Goods and services & 35.2 & 47.8 & 60.7 & 83.1 & 99.6 \\
\hline Subsidies and transfers & 19.2 & 25.2 & 28.4 & 43.5 & 55.8 \\
\hline Interest payments & 1.7 & 1.8 & 3.9 & 21.2 & 11.1 \\
\hline Capital expenditure and net lending & 50.7 & 71.7 & 81.5 & 95.0 & 119.7 \\
\hline Capital expenditure & 10.4 & 26.6 & 26.3 & 29.4 & 34.0 \\
\hline Net lending & 11.1 & 4.0 & 6.3 & -1.9 & 12.0 \\
\hline Foreign financed projects & 29.2 & 41.1 & 48.9 & 67.6 & 73.7 \\
\hline Current balance & 10.0 & 34.2 & 26.2 & 13.4 & -5.6 \\
\hline Overall balance & -36.8 & -28.6 & -48.3 & -65.1 & -102.1 \\
\hline Financing & 36.8 & 28.6 & 48.3 & 65.1 & 102.1 \\
\hline Foreign, net & 28.2 & 39.9 & 34.7 & 92.8 & 68.6 \\
\hline Project loans & 29.2 & 41.1 & 48.9 & 67.6 & 73.7 \\
\hline Cash loans & 4.2 & 16.0 & 0.0 & 43.9 & 7.5 \\
\hline Less: Amortization & 5.2 & 17.2 & 14.2 & 18.7 & 12.7 \\
\hline Domestic, net & 8.6 & -11.3 & 13.7 & -27.7 & 33.6 \\
\hline Banking system, net & 7.3 & .20 .4 & 12.3 & -25.4 & 33.6 \\
\hline \multirow[t]{2}{*}{ Nonbank } & 1.3 & 9.1 & 1.4 & -2.3 & 0.0 \\
\hline & \multicolumn{5}{|c|}{ (In percent of GDP) } \\
\hline Total revenue & 30.4 & 33.8 & 27.8 & 29.3 & 27.4 \\
\hline Current revenue & 29.0 & 31.6 & 26.6 & 27.1 & 24.8 \\
\hline Tax revenue and social security contributions & 23.8 & 24.6 & 20.6 & 21.6 & 17.6 \\
\hline Nontax revenue & $S .2$ & 7.0 & 6.0 & 5.5 & 7.2 \\
\hline Capital revenue and privatization proceeds & 0.2 & 0.9 & 0.4 & 1.4 & 1.7 \\
\hline Grants & 1.2 & 1.2 & 0.8 & 0.8 & 1.0 \\
\hline Total expenditure and net lending & 43.4 & 40.4 & 36.0 & 37.9 & 39.1 \\
\hline Current expenditure & 25.5 & 23.7 & 22.1 & 25.4 & 25.4 \\
\hline Capital expendinure and net lending & 17.9 & 16.7 & 13.9 & 12.5 & 13.7 \\
\hline Financing & 13.0 & 6.7 & 8.2 & 8.6 & 11.7 \\
\hline Foreign, net & 10.0 & 9.3 & 5.9 & 12.2 & 7.8 \\
\hline Domestic, net & 3.0 & -2.6 & 2.3 & -3.6 & 3.8 \\
\hline
\end{tabular}

Sources: Ministry of Finance; and Fund staff estimates. 
Table 17. Mongolia: General Government Revenue, 1994-98

\begin{tabular}{|c|c|c|c|c|c|}
\hline & 1994 & 1995 & 1996 & 1997 & 1998 \\
\hline & \multicolumn{5}{|c|}{ (In billions of togrogs) } \\
\hline Total revenue and grants & 86.1 & 144.9 & 162.9 & 222.5 & 240.0 \\
\hline Current sevenue & 82.2 & 135.7 & 155.9 & 206.0 & 216.8 \\
\hline Tax revenue and social security contributions & 67.5 & 105.5 & 120.9 & 164.0 & 154.0 \\
\hline Income taxes & 32.0 & 48.5 & 45.6 & 62.6 & 41.5 \\
\hline Enterprise income tax & 29.9 & 43.2 & 39.2 & 54.9 & 31.3 \\
\hline \multirow{2}{*}{$\begin{array}{l}\text { Individual income tax } \\
\text { Taxes on goods and services }\end{array}$} & 2.2 & 5.3 & 6.4 & 7.7 & 10.1 \\
\hline & 19.5 & 28.1 & 37.2 & 63.3 & 76.9 \\
\hline Sales tax & 11.3 & 16.3 & 21.5 & 38.1 & 46.1 \\
\hline On domestic goods and services & 5.8 & 7.8 & 10.7 & 19.8 & 21.1 \\
\hline On imports & 5.5 & 8.4 & 10.8 & 18.3 & 25.0 \\
\hline Excise taxes & 5.3 & 8.6 & 11.1 & 20.3 & 24.5 \\
\hline Alcobol, vodka, and tobacco & 4.6 & 5.3 & 7.3 & 12.8 & 12.7 \\
\hline Jewelry & 0.0 & 0.0 & 0.0 & 0.0 & 0.0 \\
\hline Vehicles & 0.0 & 0.0 & 0.0 & 0.5 & 2.3 \\
\hline Petroleum products & 0.7 & 3.3 & 3.9 & 7.0 & 9.5 \\
\hline \multirow{2}{*}{$\begin{array}{l}\text { Motor vehicle tax \& petrol and diesel fuel tax } \\
\text { Customs duties and import surcharges }\end{array}$} & 2.9 & 3.3 & 4.6 & 4.8 & 6.7 \\
\hline & 7.5 & 9.6 & 13.9 & 8.8 & 1.5 \\
\hline Social security contributions & 6.3 & 15.8 & 18.5 & 21.2 & 26.1 \\
\hline Other taxes & 2.1 & 3.5 & 5.7 & 8.0 & 8.0 \\
\hline Payment for use of natural resources & 0.7 & 2.1 & 3.7 & 3.6 & 2.9 \\
\hline Payment for use of timber & 0.1 & 0.2 & 0.2 & 0.3 & 0.3 \\
\hline Payment for use of land & 0.4 & 0.4 & 0.6 & 0.8 & 1.6 \\
\hline Payment for use of water & 0.0 & 0.0 & 0.1 & 0.2 & 0.1 \\
\hline Stamp tax & 0.7 & 0.6 & 0.7 & 2.1 & 1.9 \\
\hline Other taxes and fees & 0.2 & 0.2 & 0.5 & 1.0 & 1.3 \\
\hline Nontax revenue & 14.7 & 30.2 & 35.0 & 42.0 & 62.8 \\
\hline Capital revenue and privatization proceeds & 0.7 & 3.9 & 2.6 & 10.5 & 14.6 \\
\hline Grants 1/ & 3.3 & 5.1 & 4.4 & 6.0 & 8.6 \\
\hline & & (In per & total rev & & \\
\hline Current revenue & 95.4 & 87.2 & 87.2 & 92.6 & 90.3 \\
\hline Tax revenue and social security contributions & 78.3 & 53.0 & 50.8 & 73.3 & 64.2 \\
\hline Corporate tax & 34.7 & 29.9 & 24.1 & 24.7 & 13.1 \\
\hline Individual income tax & 2.5 & 3.7 & 3.9 & 3.5 & 4.2 \\
\hline Taxes on goods and services & 22.7 & 19.4 & 22.9 & 28.4 & 31.1 \\
\hline Social security contributions & 7.3 & 10.9 & 11.3 & 9.5 & 10.9 \\
\hline Other taxes & 2.4 & 2.4 & 3.5 & 3.6 & 3.3 \\
\hline Nontax revenue & 17.1 & 20.9 & 21.5 & 18.9 & 26.2 \\
\hline Capital revenue and privatization proceeds & 0.8 & 2.7 & 1.6 & 4.7 & 6.1 \\
\hline Grants & 3.8 & 3.5 & 2.7 & 2.7 & 3.6 \\
\hline & & (In & $t$ of GDF & & \\
\hline Total revenue & 30.4 & 33.8 & 27.8 & 29.3 & 27.4 \\
\hline Current revenue & 29.0 & 31.6 & 26.6 & 27.1 & 24.8 \\
\hline Tax revenue and social security contributions & 23.8 & 24.6 & 20.6 & 21.6 & 17.6 \\
\hline Enterprise income tax & 10.5 & 10.1 & 6.7 & 7.3 & 3.5 \\
\hline Individual income tax & 0.8 & 1.2 & 1.1 & 1.0 & 1.2 \\
\hline Taxes on goods and services & 6.9 & 6.5 & 6.3 & 8.3 & 8.8 \\
\hline Customs duties and import surcharges & 2.7 & 2.2 & 2.4 & 1.2 & 0.2 \\
\hline Social security contributions & 2.2 & 3.7 & 3.1 & 2.8 & 3.0 \\
\hline Other taxes & 0.7 & 0.8 & 1.0 & 1.1 & 0.9 \\
\hline Nontax revenue & 5.2 & 7.0 & 6.0 & 5.5 & 7.2 \\
\hline Capital revenue and privatization proceeds & 0.2 & 0.9 & 0.4 & 1.4 & 1.7 \\
\hline Grants & 1.2 & 1.2 & 0.8 & 0.8 & 1.0 \\
\hline
\end{tabular}

Sources: Ministry of Finance; and Fund staff estimates.

1/ Grants relate to receipt of the budget of counterpart funds from recipient of the grants. This differs from the balance of payments definition which records grants when they are received from abroad. 
Table 18. Mongolia: General Government Expenditure, 1994-98

\begin{tabular}{|c|c|c|c|c|c|}
\hline & 1994 & 1995 & 1996 & 1997 & 1998 \\
\hline & \multicolumn{4}{|c|}{ (In billions of togrogs) } & \\
\hline Total expenditure and net lending & 122.9 & 173.3 & 211.3 & 287.6 & 342.1 \\
\hline Current expenditure & 72.2 & 101.5 & 129.8 & 192.6 & 222.4 \\
\hline Wages and salaries & 16.1 & 26.8 & 36.7 & 44.8 & 56.0 \\
\hline Goods and services & 35.2 & 47.8 & 60.7 & 83.1 & 99.6 \\
\hline Subsidies and current transfers & 19.2 & 25.2 & 28.4 & 43.5 & 55.8 \\
\hline Subsidies & 5.4 & 3.6 & 0.7 & 0.4 & 0.4 \\
\hline Transfers & 13.8 & 21.6 & 27.7 & 43.2 & 55.5 \\
\hline Social Security Fund & 11.2 & 18.5 & 24.1 & 30.7 & 38.8 \\
\hline Social Assistance Fund & 1.4 & 2.1 & 3.2 & 10.0 & 10.3 \\
\hline Other transfers & 1.3 & 1.0 & 0.4 & 2.5 & 6.4 \\
\hline Interest payment & 1.7 & 1.8 & 3.9 & 21.2 & 11.1 \\
\hline Capital expenditure and net lending & 50.7 & 71.7 & 81.5 & 95.0 & 119.7 \\
\hline Capital expenditure & 10.4 & 26.6 & 26.3 & 29.4 & 34.0 \\
\hline Lending minus repayment & 11.1 & 4.0 & 6.3 & -1.9 & 12.0 \\
\hline \multirow[t]{2}{*}{ Foreign financed projects } & 29.2 & 41.1 & 48.9 & 67.6 & 73.7 \\
\hline & \multicolumn{4}{|c|}{ (In percent of total expenditure) } & \\
\hline Current expenditure & 58.7 & 58.6 & 61.4 & 67.0 & 65.0 \\
\hline Wages and salaries & 13.1 & 15.4 & 17.4 & 15.6 & 16.4 \\
\hline Goods and services & 28.6 & 27.6 & 28.7 & 28.9 & 29.1 \\
\hline Subsidies and transfers & 15.6 & 14.5 & 13.5 & 15.1 & 16.3 \\
\hline Subsidies & 4.4 & 2.1 & 0.3 & 0.1 & 0.1 \\
\hline Transfers and other & 11.2 & 12.4 & 13.1 & 15.0 . & 16.2 \\
\hline Social Security Fund & 9.1 & 10.7 & 11.4 & 10.7 & 11.3 \\
\hline Other & 2.2 & 1.8 & 1.7 & 4.3 & 4.9 \\
\hline Interest payments & 1.4 & 1.0 & 1.9 & 7.4 & 3.2 \\
\hline Capital expenditure and net lending & 41.3 & 41.4 & 38.6 & 33.0 & 35.0 \\
\hline Capital expenditure & 8.5 & 15.4 & 12.5 & 10.2 & 9.9 \\
\hline Lending minus repayment & 9.0 & 2.3 & 3.0 & -0.7 & 3.5 \\
\hline \multirow[t]{2}{*}{ Foreign financed projects } & 23.8 & 23.7 & 23.1 & 23.5 & 21.6 \\
\hline & \multicolumn{4}{|c|}{ (In percent of GDP) } & \\
\hline Total expenditure and net lending & 43.4 & 40.4 & 36.0 & 37.9 & 39.1 \\
\hline Current expenditure & 25.5 & 23.7 & 22.1 & 25.4 & 25.4 \\
\hline Wages and salaries & 5.7 & 6.2 & 6.3 & 5.9 & 6.4 \\
\hline Goods and services & 12.4 & 11.1 & 10.3 & 11.0 & 11.4 \\
\hline Subsidies and current transfers & 6.8 & 5.9 & 4.8 & 5.7 & 6.4 \\
\hline Subsidies & 1.9 & 0.8 & 0.1 & 0.0 & 0.0 \\
\hline Transfers & 4.9 & 5.0 & 4.7 & 5.7 & 6.3 \\
\hline Social Security Fund & 3.9 & 4.3 & 4.1 & 4.0 & 4.4 \\
\hline Social Assistance Fund & 0.5 & 0.5 & 0.5 & 1.3 & 1.2 \\
\hline Other transfers & 0.4 & 0.2 & 0.1 & 0.3 & 0.7 \\
\hline Interest payment & 0.6 & 0.4 & 0.7 & 2.8 & 1.3 \\
\hline Capital expenditure and net lending & 17.9 & 16.7 & 13.9 & 12.5 & 13.7 \\
\hline Capital expenditure & 3.7 & 6.2 & 4.5 & 3.9 & 3.9 \\
\hline Lending minus repayment & 3.9 & 0.9 & 1.1 & -0.3 & 1.4 \\
\hline Foreign finance projects & 10.3 & 9.6 & 8.3 & 8.9 & 8.4 \\
\hline
\end{tabular}


Table 19. Mongolia: General Government Expenditure by Function, 1994-98

\begin{tabular}{|c|c|c|c|c|c|}
\hline & 1994 & 1995 & 1996 & 1997 & 1998 \\
\hline & \multicolumn{5}{|c|}{ (In billions of togrogs) } \\
\hline Total expenditure & 122.9 & 173.3 & 211.3 & 287.6 & 342.1 \\
\hline General public services & 8.4 & 13.4 & 20.0 & 25.0 & 31.9 \\
\hline Defense & 7.0 & 9.7 & 12.1 & 15.4 & 17.7 \\
\hline Public order and safety & 4.2 & 7.3 & 10.0 & 12.0 & 15.0 \\
\hline Education & 16.4 & 25.3 & 33.7 & 46.6 & 58.4 \\
\hline Health & 11.6 & 16.9 & 22.4 & 28.6 & 32.9 \\
\hline Social security and welfare & 13.5 & 23.4 & 28.7 & 42.9 & 51.7 \\
\hline Housing and community services & 1.5 & 2.4 & 2.3 & 3.4 & 3.8 \\
\hline Recreation and culture & 3.8 & 5.3 & 6.8 & 9.3 & 11.4 \\
\hline Economic affairs & 26.8 & 27.4 & 28.0 & 23.7 & 28.6 \\
\hline \multirow[t]{2}{*}{ Other $1 /$} & 29.7 & 42.1 & 47.4 & 80.7 & 90.9 \\
\hline & \multicolumn{5}{|c|}{ (In percent of total expenditure) } \\
\hline General public services & 6.8 & 7.7 & 9.5 & 8.7 & 9.3 \\
\hline Defense & 5.7 & 5.6 & 5.7 & 5.4 & 5.2 \\
\hline Public order and safety & 3.2 & 4.1 & 4.6 & 4.6 & 4.6 \\
\hline Education & 12.4 & 13.6 & 14.8 & 14.8 & 14.8 \\
\hline Health & 9.4 & 9.8 & 10.6 & 9.9 & 9.6 \\
\hline Social security and welfare & 10.9 & 13.5 & 13.6 & 14.9 & 15.1 \\
\hline Housing and community services & 1.2 & 1.4 & 1.1 & 1.2 & 1.1 \\
\hline Recreation and culture & 3.0 & 2.9 & 3.1 & 3.1 & 3.1 \\
\hline Economic affairs & 21.8 & 15.8 & 13.2 & 8.2 & 8.4 \\
\hline \multirow[t]{2}{*}{ Other $1 /$} & 24.1 & 24.3 & 22.4 & 28.1 & 26.6 \\
\hline & \multicolumn{5}{|c|}{ (In percent of GDP) } \\
\hline Total expenditure & 43.4 & 40.4 & 36.0 & 37.9 & 39.1 \\
\hline General public services & 3.0 & 3.1 & 3.4 & 3.3 & 3.6 \\
\hline Defense & 2.5 & 2.3 & 2.1 & 2.0 & 2.0 \\
\hline Public order and safety & 1.5 & 1.7 & 1.7 & 1.6 & 1.7 \\
\hline Education & 5.8 & 5.9 & 5.7 & 6.1 & 6.7 \\
\hline Health & 4.1 & 3.9 & 3.8 & 3.8 & 3.8 \\
\hline Social security and welfare & 4.8 & 5.5 & 4.9 & 5.6 & 5.9 \\
\hline Housing and community services & 0.5 & 0.6 & 0.4 & 0.4 & 0.4 \\
\hline Recreation and culture & 1.4 & 1.2 & 1.2 & 1.2 & 1.3 \\
\hline Economic affairs & 9.4 & 6.4 & 4.8 & 3.1 & 3.3 \\
\hline Other $1 /$ & 10.5 & 9.8 & 8.1 & 10.6 & 10.4 \\
\hline
\end{tabular}

Sources: Ministry of Finance; and Fund staff estimates.

1/ Includes capital expenditure and net lending (foreign financed projects). 
Table 20. Mongolia: General Government Social Expenditure, 1994-98

\begin{tabular}{|c|c|c|c|c|c|}
\hline & 1994 & 1995 & 1996 & 1997 & 1998 \\
\hline & \multicolumn{5}{|c|}{ (In billions of togrogs) } \\
\hline Total expenditure & 122.9 & 173.3 & 211.3 & 287.6 & 342.1 \\
\hline Current expenditure & 72.2 & 101.5 & 129.8 & 192.6 & 222.4 \\
\hline Social expenditure & 46.8 & 73.3 & 93.8 & 130.8 & 158.2 \\
\hline Social security and social assistance & 13.5 & 23.4 & 28.7 & 42.9 & 51.7 \\
\hline Education & 16.4 & 25.3 & 33.7 & 46.6 & 58.4 \\
\hline Health & 11.6 & 16.9 & 22.4 & 28.6 & 32.9 \\
\hline Housing and community & 1.5 & 2.4 & 2.3 & 3.4 & 3.8 \\
\hline \multirow[t]{2}{*}{ Recreational, cultural affairs and services } & 3.8 & 5.3 & 6.8 & 9.3 & 11.4 \\
\hline & \multicolumn{5}{|c|}{ (In percent of total expenditure) } \\
\hline Social expenditure & 38.1 & 42.3 & 44.4 & 45.5 & 46.2 \\
\hline Social security and social assistance & 10.9 & 13.5 & 13.6 & 14.9 & 15.1 \\
\hline Education & 13.4 & 14.6 & 15.9 & 16.2 & 17.1 \\
\hline Health & 9.4 & 9.8 & 10.6 & 9.9 & 9.6 \\
\hline Housing and community & 1.2 & 1.4 & 1.1 & 1.2 & 1.1 \\
\hline Recreational, cultural affairs and services & 3.1 & 3.0 & 3.2 & 3.2 & 3.3 \\
\hline
\end{tabular}

Sources: Ministry of Finance; and Fund staff estimates. 
Table 21. Mongolia: Subsidies and Transfers, 1994-98

\begin{tabular}{|c|c|c|c|c|c|}
\hline & 1994 & 1995 & 1996 & 1997 & 1998 \\
\hline & \multicolumn{5}{|c|}{ (In billions of togrogs) } \\
\hline Total subsidies and transfers & 23.9 & 31.7 & 31.3 & 43.2 & 55.9 \\
\hline Total subsidies & 9.8 & 9.8 & 0.7 & 0.4 & 0.4 \\
\hline Energy & 4.1 & 3.1 & 0.4 & 0.1 & 0.1 \\
\hline Mapping office & 0.1 & 0.1 & 0.1 & 0.1 & 0.1 \\
\hline Urban transport & 1.3 & 0.5 & 0.3 & 0.3 & 0.3 \\
\hline Veterinary services $1 /$ & 1.3 & 2.2 & 0.0 & 0.0 & $\ldots$ \\
\hline Medicines 1/ & 3.0 & 3.9 & 0.0 & 0.0 & $\cdots$ \\
\hline Total transfers & 13.9 & 21.6 & 27.7 & 43.2 & 55.5 \\
\hline Social Security Fund & 11.2 & 18.5 & 24.1 & 30.7 & 38.8 \\
\hline Social Assistance Fund & 1.4 & 2.1 & 3.2 & 10.0 & 10.3 \\
\hline \multirow[t]{2}{*}{ Other } & 1.3 & 1.0 & 0.4 & 2.5 & 6.4 \\
\hline & \multicolumn{5}{|c|}{ (In percent of GDP) } \\
\hline Total subsidies and transfers & 8.4 & 7.4 & 5.3 & 5.7 & 6.4 \\
\hline Total subsidies & 3.4 & 2.3 & 0.1 & 0.1 & 0.1 \\
\hline Total transfers & 4.9 & 5.0 & 4.7 & 5.7 & 6.3 \\
\hline
\end{tabular}

Sources: Ministry of Finance; and Fund staff estimates.

1/ Not included in the general government accounts. 
Table 22. Mongolia: Social Security and Social Assistance Funds 1994-98 1/

\begin{tabular}{|c|c|c|c|c|c|}
\hline & 1994 & 1995 & 1996 & 1997 & 1998 \\
\hline & \multicolumn{5}{|c|}{ (In billions of togrogs) } \\
\hline \multicolumn{6}{|l|}{ Social Security Fund } \\
\hline Total revenue & 13.82 & 23.09 & 27.86 & 33.82 & 38.53 \\
\hline Budget transfers & 7.59 & 7.35 & 6.58 & 5.00 & 8.56 \\
\hline Payroll tax $2 /$ & 6.21 & 15.51 & 20.83 & 28.55 & 29.47 \\
\hline Civil servants & 2.40 & 3.38 & 4.87 & 7.75 & 8.66 \\
\hline Business entities & 3.82 & 7.50 & 9.87 & 12.79 & 12.39 \\
\hline Employees & $\ldots$ & 4.63 & 6.08 & 8.01 & 8.41 \\
\hline Other $3 /$ & 0.02 & 0.23 & 0.46 & 0.27 & 0.50 \\
\hline Total expenditure & 13.20 & 18.97 & 25.36 & 31.87 & 40.02 \\
\hline Old age pension & 8.61 & 13.91 & 18.10 & 22.53 & 26.54 \\
\hline Handicapped persons & 0.87 & 1.53 & 2.00 & 2.45 & 3.26 \\
\hline Survivors' benefits & 1.25 & 2.15 & 2.58 & 3.58 & 4.45 \\
\hline Disability benefits & 0.44 & 0.46 & 0.52 & 0.52 & 0.65 \\
\hline Death benefits & $\ldots$ & 0.40 & 0.69 & 0.59 & 0.71 \\
\hline Allowance for accidents & $\ldots$ & 0.02 & 0.18 & 0.81 & 1.01 \\
\hline Serviceman's pension & $\ldots$ & $\ldots$ & $\ldots$ & $\ldots$ & 1.85 \\
\hline Unemployment benefits & $\ldots$ & $\ldots$ & $\ldots$ & 0.20 & 0.30 \\
\hline Administration & 0.35 & 0.48 & 1.20 & 0.93 & 0.88 \\
\hline Capital investment & 0.04 & 0.03 & 0.02 & 0.26 & 0.36 \\
\hline Other $3 /$ & 1.65 & $\ldots$ & 0.09 & $\cdots$ & 0.00 \\
\hline Overall balance & 0.62 & 4.12 & 2.50 & 1.95 & -1.49 \\
\hline \multicolumn{6}{|l|}{ Social Assistance Fund } \\
\hline Total expenditure & $\ldots$ & 1.78 & 3.21 & 10.29 & 7.40 \\
\hline Pregnancy & $\ldots$ & 0.43 & 0.66 & 1.76 & 1.88 \\
\hline Mothers with large families & $\ldots$ & 0.10 & 0.08 & 0.50 & 0.47 \\
\hline Child care & $\ldots$ & 0.24 & 0.66 & 1.92 & 0.07 \\
\hline Twins & $\ldots$ & $\ldots$ & 0.00 & 0.00 & 0.00 \\
\hline Infanct nursing & $\ldots$ & 0.03 & 0.06 & 0.04 & 1.76 \\
\hline Social pension & $\ldots$ & 0.51 & 1.03 & 1.53 & 1.59 \\
\hline War veterans & $\ldots$ & $\ldots$ & $\ldots$ & 0.09 & 1.12 \\
\hline Rest homes & $\ldots$ & 0.03 & 0.03 & 0.04 & 0.00 \\
\hline \multirow[t]{2}{*}{ Other } & $\ldots$ & 0.44 & 0.69 & 4.41 & 0.50 \\
\hline & \multicolumn{5}{|c|}{ (In percent of total govemment expenditure) } \\
\hline \multicolumn{6}{|l|}{ Memoranđum items: } \\
\hline Social Security Fund expenditure & 10.7 & 10.9 & 12.0 & 11.1 & 11.7 \\
\hline Budget transfers to the Social Security Fund & 6.2 & 4.2 & 3.1 & 1.7 & 2.5 \\
\hline \multirow[t]{2}{*}{ Social Assistance Fund expenditure } & $\ldots$ & 1.0 & 1.5 & 3.6 & 2.2 \\
\hline & \multicolumn{5}{|c|}{ (In percent of GDP) } \\
\hline Social Security Fund expenditure & 4.7 & 4.4 & 4.3 & 4.2 & 4.6 \\
\hline Budget transfers to the Social Security Fund & 2.7 & 1.7 & 1.1 & 0.7 & 1.0 \\
\hline Social Assistance Fund expenditure & $\ldots$ & 0.4 & 0.5 & 1.4 & 0.8 \\
\hline
\end{tabular}

Sources: Ministry of Finance; and Fund staff estimates.

1/ From 1995 onwards, the classifications reflect the separation of social security and social assistance payments.

2/ Starting in 1995, corresponds to Social Security contributions.

3/ Until 1995, included in allowance and benefits. 
Table 23. Mongolia: Number of Social Security Beneficiaries, 1994-98

(In thousands)

\begin{tabular}{lrrrrr}
\hline & 1994 & 1995 & 1996 & 1997 & 1998 \\
& & & & & \\
& 395.6 & 380.3 & 361.9 & 310.7 & 294.9 \\
Social security & & & & & \\
& 227.4 & 222.8 & 222.7 & 174.3 & 167.7 \\
Old age & 25.2 & 25.9 & 27.9 & 24.4 & 26.6 \\
Handicapped persons & 35.1 & 35.5 & 36.7 & 34.5 & 34.2 \\
Survivors' benefits & 89.7 & 76.4 & 55.7 & 57.7 & 56.7 \\
Disability benefits & 18.2 & 19.7 & 18.9 & 19.8 & 9.7 \\
Other benefits & & & & & \\
& 179.8 & 282.3 & 349.8 & 530.0 & 454.6 \\
Social assistance & 29.4 & 15.2 & 27.0 & 70.8 & 69.0 \\
Pregnancy & 63.5 & 170.7 & 238.3 & 294.4 & 233.4 \\
Mothers with large families & 53.8 & 1.6 & 3.2 & 93.5 & 5.8 \\
Child care & 0.1 & 0.1 & 0.2 & 0.4 & 0.6 \\
Twins & 6.3 & 52.8 & 28.7 & 4.3 & 40.3 \\
Infant nursing & 9.0 & 9.0 & 18.2 & 40.7 & 57.6 \\
War veterans & $\ldots$ & 20.3 & 22.9 & 23.2 & 24.8 \\
Social pension & $\ldots$ & $\ldots$ & $\ldots$ & $\ldots$ & 5.2 \\
Disabled Nursing homes for elderly & 17.7 & 12.6 & 11.3 & 2.7 & 17.9 \\
Other & & & & &
\end{tabular}

Sources: Ministry of Finance; and Fund staff estimates. 
Table 24. Mongolia: Government Staff and Wage Bill, 1995-98

\begin{tabular}{|c|c|c|c|c|}
\hline & 1995 & 1996 & 1997 & 1998 \\
\hline & \multicolumn{4}{|c|}{ (Number of positions) } \\
\hline \multicolumn{5}{|l|}{ Government staff $1 /$} \\
\hline General public services & 12,951 & 13,690 & 15,780 & 15,515 \\
\hline Public order and safety & 15,287 & 13,806 & 12,891 & 13,995 \\
\hline Education & 59,081 & 50,686 & 47,686 & 47,649 \\
\hline Health & 35,100 & 30,074 & 27,266 & 27,021 \\
\hline Social security and welfare & 1,194 & 1,111 & 1,591 & 1,571 \\
\hline Recreation and culture & 6,291 & 6,747 & 6,303 & 6,307 \\
\hline Agriculture and forestry & 3,936 & 3,720 & 3,239 & 1,423 \\
\hline Other services & 17,653 & 22,062 & 22,057 & 21,711 \\
\hline \multirow[t]{2}{*}{ Total } & 151,493 & 141,896 & 136,813 & 135,192 \\
\hline & \multicolumn{4}{|c|}{ (In billions of togrogs) } \\
\hline \multicolumn{5}{|l|}{ Wages and salaries } \\
\hline General public services & 3.4 & 4.9 & 6.0 & 8.3 \\
\hline Public order and safety & 2.7 & 4.0 & 4.6 & 6.4 \\
\hline Education & 9.9 & 13.7 & 16.8 & 20.6 \\
\hline Health & 5.2 & 6.7 & 8.9 & 9.6 \\
\hline Social security and welfare & 0.3 & 0.5 & 0.6 & 0.6 \\
\hline Recreation and culture & 1.0 & 1.4 & 2.2 & 2.1 \\
\hline Agriculture and forestry & 0.7 & 0.9 & 1.2 & 0.6 \\
\hline Other services & 3.5 & 4.7 & 4.4 & 7.6 \\
\hline Total & 26.8 & 36.7 & 44.8 & 56.0 \\
\hline
\end{tabular}

Sources: Ministry of Finance; and Fund staff estimates.

1/ Number of official positions. The decline in numbers mainly reflects more accurate recording and the elimination of vacancies. 
Table 25. Mongolia: Expenditure of the Health Sector, 1995-98

(In billions of togrogs)

\begin{tabular}{lcccr}
\hline & 1995 & 1996 & 1997 & 1998 \\
& & & & \\
& 16.9 & 22.4 & 28.6 & 32.9 \\
Total expenditure & & & & \\
& 5.2 & 6.7 & 7.8 & 9.6 \\
Wages and salaries & & & & \\
& 1.0 & 1.3 & 2.0 & 2.4 \\
Social security & 0.8 & 1.1 & 1.7 & 2.0 \\
Social security payments & 0.2 & 0.2 & 0.3 & 0.5 \\
Health insurance & & & & 19.3 \\
& 10.0 & 13.2 & 17.3 & 1.1 \\
Other goods and services & 0.5 & 1.0 & 1.2 & 3.5 \\
Electricity & 1.8 & 2.8 & 3.3 & 1.2 \\
Fuel and heating & 0.9 & 0.9 & 1.2 & 2.0 \\
Transport (fuel) & 1.2 & 1.7 & 2.0 & 5.7 \\
Food & 2.8 & 3.6 & 4.8 & 5.8 \\
Medicines & 2.7 & 3.2 & 4.7 & 1.1 \\
Other & & & & 0.5 \\
Investment & 0.6 & 0.9 & 1.0 & \\
Capital repair expenditures & & & & \\
& 0.2 & 0.3 & 0.5 & \\
\hline & & & & \\
\hline
\end{tabular}

Sources: Ministry of Finance; and Fund staff estimates. 
Table 26. Mongolia: Expenditure of the Education Sector, 1994-98

(In billions of togrogs)

\begin{tabular}{|c|c|c|c|c|c|}
\hline & 1994 & 1995 & 1996 & 1997 & 1998 \\
\hline Total expenditure & 16.44 & 25.33 & 33.67 & 46.64 & 58.36 \\
\hline Wages and salaries & 5.84 & 9.92 & 13.70 & 16.42 & 20.64 \\
\hline Social security & 0.89 & 1.80 & 2.48 & 3.97 & 4.46 \\
\hline Social security payments & 0.74 & 1.49 & 2.04 & 3.25 & 3.60 \\
\hline Health insurance & 0.15 & 0.31 & 0.44 & 0.72 & 0.86 \\
\hline Other goods and services & 9.39 & 12.53 & 16.01 & 24.67 & 30.08 \\
\hline Electricity & 0.62 & 0.80 & 1.27 & 1.75 & 1.85 \\
\hline Fuel and heating & 3.96 & 4.83 & 6.45 & 9.16 & 10.26 \\
\hline Transport (fuel) & 0.32 & 0.35 & 0.41 & 0.48 & 0.45 \\
\hline Food & 1.18 & 1.92 & 2.39 & 3.31 & 3.32 \\
\hline Medicines & 0.02 & 0.03 & 0.03 & 0.01 & 0.01 \\
\hline Other & 3.29 & 4.60 & 5.46 & 9.95 & 14.18 \\
\hline \multirow[t]{2}{*}{ Investment } & 0.21 & 0.53 & 0.85 & 0.73 & 1.78 \\
\hline & 0.00 & 0.00 & 0.00 & 0.00 & 0.00 \\
\hline Capital repair expenditures & 0.11 & 0.55 & 0.63 & 0.87 & 1.41 \\
\hline Lending minus repayment & $\ldots$ & $\ldots$ & $\ldots$ & $\ldots$ & 0.02 \\
\hline
\end{tabular}

Sources: Ministry of Finance; and Fund staff estimates. 
Table 27. Mongolia: Budgetary Support for Enterprises, 1996-98

(In billions of togrogs)

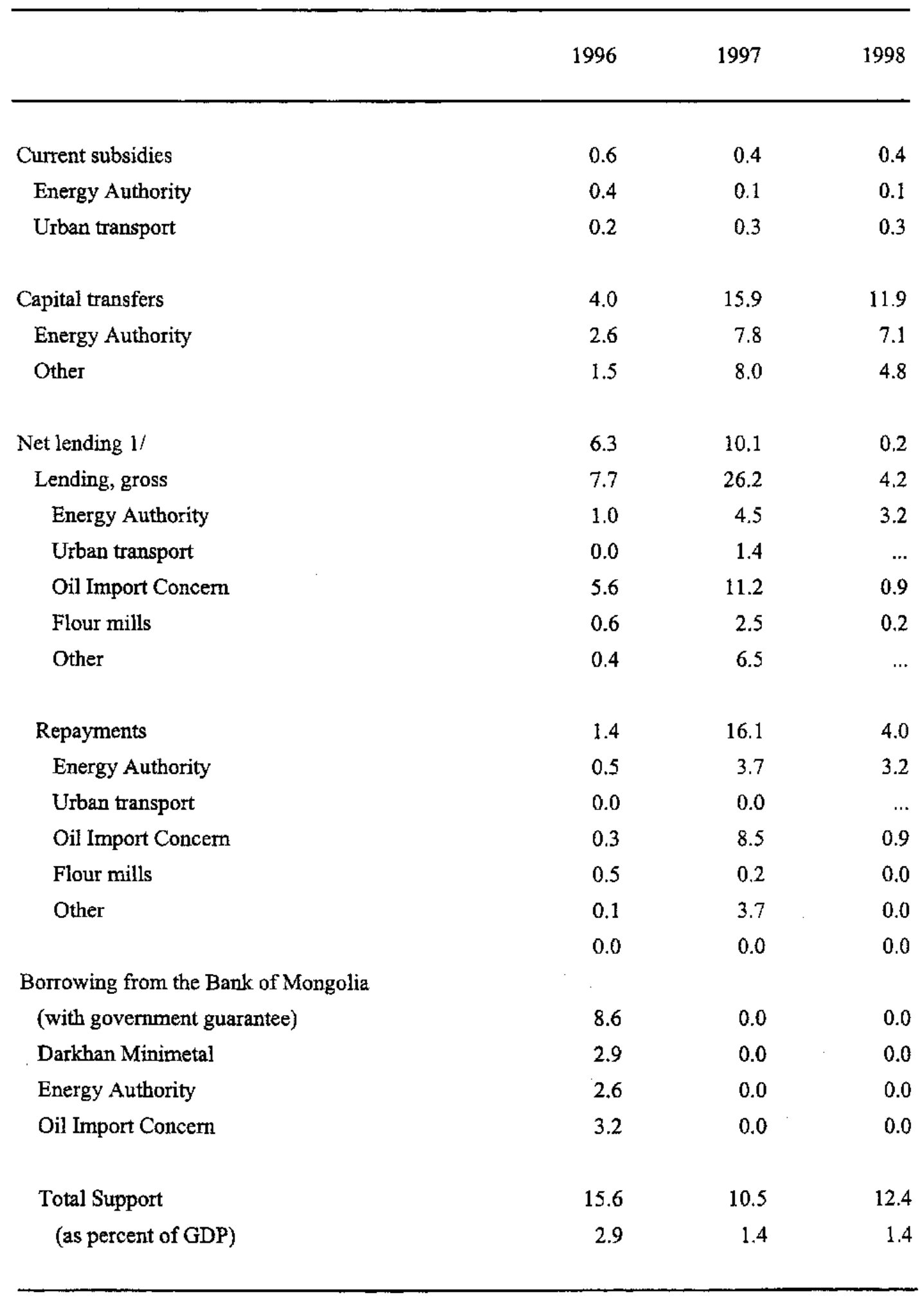

Sources: Ministry of Finance; and Fund staff estimates. 
Table 28. Mongolia: Monetary Survey, 1996-99

\begin{tabular}{|c|c|c|c|c|c|c|c|c|c|}
\hline & \multirow{2}{*}{$\begin{array}{l}1996 \\
\text { Dec. }\end{array}$} & \multirow{2}{*}{$\begin{array}{l}1997 \\
\text { Dec. }\end{array}$} & \multicolumn{4}{|c|}{1998} & \multicolumn{3}{|c|}{1999} \\
\hline & & & Mar. & June & Sep. & Dec. & Mar. & June & Sep. \\
\hline & \multicolumn{9}{|c|}{ (In billions of togrogs) } \\
\hline Broad Money & 128.4 & 170.1 & 153.6 & 160.2 & 166.0 & 167.3 & 153.7 & 184.6 & 199.2 \\
\hline Currency & 41.7 & 49.8 & 41.5 & 55.4 & 54.0 & 56.4 & 48.1 & 73.8 & 76.5 \\
\hline Deposits & 86.7 & 120.3 & 112.1 & 104.8 & 112.0 & 110.8 & 105.6 & 110.8 & 122.7 \\
\hline Demand deposits & 22.6 & 26.3 & 22.3 & 23.3 & 25.2 & 26.1 & 18.2 & 18.8 & 24.3 \\
\hline Togrog time deposits & 35.2 & 44.7 & 49.9 & 43.5 & 42.2 & 44.8 & 42.9 & 41.2 & 44.0 \\
\hline Forex time deposits & 28.9 & 49.3 & 40.0 & 38.0 & 44.6 & 39.8 & 44.5 & 50.9 & 54.4 \\
\hline Net foreign assets & 66.7 & 112.3 & 80.8 & 69.0 & 76.7 & 76.8 & 63.7 & 76.1 & 110.4 \\
\hline Net international reserves & 74.4 & 131.0 & 95.7 & 84.7 & 91.7 & 93.6 & 83.4 & 94.4 & 122.9 \\
\hline Bank of Mongolia & 37.6 & 73.1 & 60.1 & 57.5 & 60.4 & 67.5 & 54.0 & 62.3 & 89.7 \\
\hline Commercial banks & 36.8 & 57.9 & 35.5 & 27.3 & 31.3 & 26.1 & 29.3 & 32.1 & 33.1 \\
\hline Other NFA $1 /$ & -7.7 & -18.8 & -14.9 & -15.7 & -15.0 & -16.8 & -19.7 & -18.3 & -12.5 \\
\hline Bank of Mongolia & -5.5 & -1.7 & 3.2 & 1.6 & 3.8 & 3.1 & 1.8 & 3.0 & 4.2 \\
\hline Commercial banks & -2.2 & -17.1 & -18.1 & -17.3 & -18.8 & -19.9 & -21.5 & -21.3 & -16.7 \\
\hline Net domestic assets & 61.7 & 57.8 & 72.8 & 91.2 & 89.3 & 90.4 & 90.0 & 108.5 & 88.8 \\
\hline Domestic credit & 96.7 & 91.7 & 114.3 & 128.4 & 138.4 & 146.8 & 163.5 & 173.9 & 150.5 \\
\hline Net credit to govemment & 20.5 & 21.3 & 29.1 & 33.4 & 41.0 & 47.9 & 58.1 & 68.7 & 44.0 \\
\hline Credit & 51.5 & 65.9 & 61.4 & 64.8 & 70.1 & 70.9 & 83.8 & 95.8 & 78.1 \\
\hline Minus: Deposits & 31.0 & 44.5 & 32.3 & 31.5 & 29.1 & 23.0 & 25.7 & 27.1 & 34.0 \\
\hline Claims on nonbanks & 76.2 & 70.4 & 85.2 & 95.0 & 97.4 & 98.9 & 105.4 & 105.2 & 106.4 \\
\hline Claims on public enterprises & 18.6 & 11.7 & 15.6 & 17.2 & 21.0 & 18.3 & 18.0 & 14.6 & 15.9 \\
\hline Claims on the private sector & 30.8 & 44.2 & 51.6 & 55.0 & 51.6 & 48.0 & 51.9 & 51.3 & 44.9 \\
\hline Nonperforming loans & 26.8 & 14.5 & 18.0 & 22.8 & 24.8 & 32.6 & 35.5 & 39.3 & 45.6 \\
\hline \multirow[t]{2}{*}{ Other items, net } & -35.0 & -33.9 & -41.5 & -37.2 & -49.1 & -56.4 & -73.5 & -65.4 & -61.6 \\
\hline & \multicolumn{9}{|c|}{ (In percent) } \\
\hline \multirow{2}{*}{\multicolumn{10}{|c|}{$\begin{array}{l}\text { Memorandum items: } \\
\text { Twleve-month percentage change }\end{array}$}} \\
\hline & & & & & & & & & \\
\hline Broad money $1 /$ & 25.8 & 19.8 & 34.2 & 16.2 & 9.5 & 8.8 & 0.0 & 15.2 & 20.0 \\
\hline Net foreign assets & 69.0 & 68.3 & 7.3 & -6.2 & -16.2 & -31.6 & -21.2 & 10.3 & 44.0 \\
\hline Domestic credit & 133.4 & -5.1 & 20.0 & 35.7 & 56.5 & 60.1 & 43.1 & 35.5 & 8.7 \\
\hline Claims on public enterprises & 17.2 & -36.9 & 10.1 & 22.7 & 97.4 & 56.2 & 15.7 & -14.9 & -24.2 \\
\hline Claims on the private sector & -33.0 & 43.3 & 62.4 & 73.0 & 48.0 & 8.5 & 0.4 & -6.9 & -13.1 \\
\hline \multicolumn{10}{|l|}{ Key ratios } \\
\hline Currency/total deposits & 48.1 & 41.4 & 37.0 & 52.8 & 48.2 & 50.9 & 45.5 & 66.6 & 62.4 \\
\hline Demand deposits/total deposits & 26.1 & 21.9 & 19.9 & 22.2 & 22.5 & 23.6 & 17.3 & 17.0 & 19.8 \\
\hline Togrog time deposits/total deposits & 40.6 & 37.1 & 44.5 & 41.5 & 37.7 & 40.5 & 40.6 & 37.1 & 35.9 \\
\hline Forex time deposits/total deposits & 33.4 & 41.0 & 35.7 & 36.3 & 39.8 & 35.9 & 42.2 & 45.9 & 44.3 \\
\hline Currency/broad money & 32.5 & 29.3 & 27.0 & 34.6 & 32.5 & 33.7 & 31.3 & 40.0 & 38.4 \\
\hline Demand deposits/broad money & 17.6 & 15.5 & 14.5 & 14.5 & 15.2 & 15.6 & 11.9 & 10.2 & 12.2 \\
\hline Time depsoits/broad money & 49.9 & 55.2 & 58.5 & 50.9 & 52.3 & 50.6 & 56.9 & 49.8 & 49.4 \\
\hline
\end{tabular}

Sources: Bank of Mongolia; and Fund staff estimates.

1/ The end-1997 broad money stock is artificially inflated by a one-off increase due to a US\$20 million (Tog 16.3 billion) deposit, which was reversed in early 1998 . The annual broad money growth rate reflects an adjustment for this increase. 
Table 29. Mongolia: Balance Sheet of the Bank of Mongolia, 1996-99

\begin{tabular}{|c|c|c|c|c|c|c|c|c|c|}
\hline & \multirow{2}{*}{$\begin{array}{l}1996 \\
\text { Dec. }\end{array}$} & \multirow{2}{*}{$\begin{array}{l}1997 \\
\text { Dec. }\end{array}$} & \multicolumn{4}{|c|}{1998} & \multicolumn{3}{|c|}{1999} \\
\hline & & & Mar. & June & Sep. & Dec. & Mar. & June & Sep. \\
\hline & \multicolumn{9}{|c|}{ (In billions of togrogs) } \\
\hline Reserve money & 51.2 & 64.6 & 58.2 & 72.0 & 74.5 & 73.3 & 60.0 & 90.4 & 97.7 \\
\hline Currency outside Banks & 41.7 & 49.8 & 41.5 & 55.4 & 54.0 & 56.4 & 48.1 & 73.8 & 76.5 \\
\hline DMB reserves & 9.5 & 13.2 & 16.3 & 15.9 & 18.7 & 16.0 & 11.6 & 16.4 & 21.0 \\
\hline Cash in vaults & 4.3 & 7.0 & 5.9 & 5.9 & 5.7 & 5.0 & 4.5 & 4.6 & 6.5 \\
\hline Demand deposits of DMBs & 5.1 & 6.2 & 10.4 & 10.0 & 13.0 & 11.0 & 7.1 & 11.8 & 14.5 \\
\hline Deposits of business units & 0.0 & 1.6 & 0.4 & 0.7 & 1.8 & 0.8 & 0.3 & 0.2 & 0.2 \\
\hline Net Foreign Assets & 32.0 & 71.4 & 63.4 & 59.0 & 64.1 & 70.6 & 55.8 & 65.2 & 93.9 \\
\hline Net international reserves & 37.6 & 73.1 & 60.1 & 57.5 & 60.4 & 67.5 & 54.0 & 62.3 & 89.7 \\
\hline Assets & 67.8 & 111.8 & 102.3 & 98.9 & 103.8 & 111.1 & 102.6 & 115.5 & 146.1 \\
\hline Minus: Liabilities & 30.2 & 38.7 & 42.2 & 41.5 & 43.5 & 43.6 & 48.5 & 53.3 & 56.4 \\
\hline Other assets, net & -5.5 & -1.7 & 3.2 & 1.6 & 3.8 & 3.1 & 1.8 & 3.0 & 4.2 \\
\hline Assets & 0.0 & 2.0 & 3.2 & 1.6 & 3.8 & 3.1 & 1.8 & 3.0 & 4.2 \\
\hline Minus: Liabilities & 5.5 & 3.7 & 0.0 & 0.0 & 0.0 & 0.0 & 0.0 & 0.0 & 0.0 \\
\hline Net Domestic Assets & 19.1 & -6.9 & -5.1 & 13.0 & 10.4 & 2.7 & 4.1 & 25.2 & 3.8 \\
\hline Net Credit to Government & 30.6 & 17.6 & 24.3 & 24.2 & 26.3 & 29.7 & 33.8 & 41.7 & 29.5 \\
\hline Credit & 39.0 & 30.4 & 29.9 & 29.9 & 32.0 & 32.6 & 40.9 & 46.9 & 35.9 \\
\hline Reconstruction bonds & 15.5 & 13.8 & 13.8 & 13.8 & 19.6 & 19.6 & 19.6 & 19.6 & 19.6 \\
\hline Treasury IMF account & 0.0 & 6.4 & 6.4 & 6.4 & 6.4 & 6.4 & 6.4 & 13.2 & 13.2 \\
\hline Other & 23.5 & 10.2 & 9.7 & 9.7 & 6.0 & 6.5 & 14.8 & 14.1 & 3.1 \\
\hline Minus: Deposits & 8.4 & 12.8 & 5.7 & 5.8 & 5.8 & 2.9 & 7.0 & 5.2 & 6.3 \\
\hline Domestic currency & 4.6 & 4.8 & 4.7 & 5.2 & 2.7 & 2.5 & 5.8 & 5.1 & 6.3 \\
\hline Foreign currency & 3.8 & 6.8 & 1.0 & 0.5 & 3.1 & 0.4 & 1.3 & 0.1 & 0.1 \\
\hline Claims on deposit money banks & 1.7 & 3.1 & 3.2 & 3.2 & 7.4 & 5.6 & 8.0 & 10.3 & 7.0 \\
\hline Claims on nonbanks & 5.5 & 3.9 & 6.8 & 5.5 & 8.9 & 9.0 & 10.3 & 10.1 & 10.4 \\
\hline Minus: Central bank bills (net) & 0.0 & 19.1 & 22.6 & 9.4 & 12.1 & 11.7 & 5.2 & 8.1 & 16.3 \\
\hline Other items, net & -18.7 & -12.4 & -16.8 & -10.5 & -20.0 & -29.8 & -42.8 & -28.9 & -26.9 \\
\hline & & & & & ercent) & & & & \\
\hline \multicolumn{10}{|l|}{ Memorandum items: } \\
\hline Reserve money growth (12 month percent change) & 36.2 & 26.2 & 25.9 & 14.1 & 12.0 & 13.5 & 3.0 & 25.6 & 31.0 \\
\hline Broad money/reserve money (ratio) & 2.51 & 2.63 & 2.64 & 2.23 & 2.23 & 2.28 & 2.56 & 2.04 & 2.04 \\
\hline
\end{tabular}

Sources: Bank of Mongolia; and Fund staff estimates. 
Table 30. Mongolia: Consolidated Balance Sheet of Commercial Banks, 1996-99

\begin{tabular}{|c|c|c|c|c|c|c|c|c|c|}
\hline & \multirow{2}{*}{$\begin{array}{l}1996 \\
\text { Dec. }\end{array}$} & \multirow{2}{*}{$\begin{array}{r}1997 \\
\text { Dec. }\end{array}$} & \multicolumn{4}{|c|}{1998} & \multicolumn{3}{|c|}{1999} \\
\hline & & & Mar. & June & Sep. & $\overline{\text { Dec. }}$ & Mar. & June & Sep. \\
\hline & \multicolumn{9}{|c|}{ (In billions of togrogs) } \\
\hline ASSETS & 97.6 & 121.1 & 112.9 & 105.7 & 117.7 & $\$ 13.1$ & 107.9 & 112.8 & 125.1 \\
\hline Net foreign assets & 34.7 & 40.8 & 17.5 & 10.0 & 12.5 & 6.2 & 7.9 & 10.9 & 16.5 \\
\hline Net international reserves & 36.8 & 57.9 & 35.5 & 27.3 & 31.3 & 26.1 & 29.3 & 32.1 & 33.1 \\
\hline Assets & 43.3 & 64.9 & 35.5 & 27.3 & 31.3 & 26.1 & 30.3 & 33.3 & 34.4 \\
\hline Minus: Liabilities & 6.4 & 7.0 & 0.0 & 0.0 & 0.0 & 0.0 & 0.9 & 1.2 & 1.3 \\
\hline Other foreign assets, net & -2.2 & -17.1 & -18.1 & -17.3 & -18.8 & -19.9 & -21.5 & -21.3 & -16.7 \\
\hline Assets & 0.0 & 0.0 & 0.0 & 0.0 & 0.0 & 0.0 & 0.0 & 0.0 & 0.0 \\
\hline Minus: Liabilities & 2.2 & 17.1 & 18.1 & 17.3 & 18.8 & 19.9 & 21.5 & 21.3 & 16.7 \\
\hline Net domestic assets & 62.9 & 80.2 & 95.5 & 95.7 & 105.1 & 106.9 & 100.0 & 101.9 & 108.6 \\
\hline Reserves & 9.5 & 13.2 & 16.3 & 15.9 & 18.7 & 16.0 & 11.6 & 16.4 & 21.0 \\
\hline Cash & 4.3 & 7.0 & 5.9 & 5.9 & 5.7 & 5.0 & 4.5 & 4.6 & 6.5 \\
\hline Deposits with BOM & 5.1 & 6.2 & 10.4 & 10.0 & 13.0 & 11.0 & 7.1 & 11.8 & 14.5 \\
\hline Central bank bills & 0.0 & 19.1 & 22.6 & 9.4 & 12.1 & 11.7 & 5.2 & 8.1 & 16.3 \\
\hline Domestic credit & 60.6 & 54.1 & 78.9 & 94.4 & 99.0 & 103.9 & 115.1 & 117.8 & 106.2 \\
\hline Net credit to govemment & -10.1 & 3.7 & 4.9 & 9.2 & 14.7 & 18.2 & 24.3 & 27.0 & 14.5 \\
\hline Claims on Government & 12.5 & 35.5 & 31.5 & 34.9 & 38.1 & 38.3 & 43.0 & 48.9 & 42.2 \\
\hline Less: Government deposits & 22.6 & 31.7 & 26.6 & 25.7 & 23.3 & 20.1 & 18.6 & 22.0 & 27.7 \\
\hline Loans to nonbanks & 70.7 & 50.4 & 74.0 & 85.2 & 84.2 & 85.6 & 90.8 & 90.9 & 91.7 \\
\hline Public enterprises & 13.1 & 7.8 & 8.7 & 11.6 & 12.1 & 9.3 & 7.7 & 4.6 & 5.5 \\
\hline Private sector & 30.8 & 28.1 & 47.3 & 50.7 & 47.3 & 43.7 & 47.6 & 47.0 & 40.6 \\
\hline Nonperforming loans & 26.8 & 14.5 & 18.0 & 22.8 & 24.8 & 32.6 & 35.5 & 39.3 & 45.6 \\
\hline Other assets, net & -7.2 & -6.1 & -22.3 & -24.0 & -24.6 & -24.7 & -31.8 & -40.4 & -34.9 \\
\hline LIABILITIES & 97.6 & 121.1 & 112.9 & 105.7 & 117.7 & 113.1 & 107.9 & 112.8 & 125.1 \\
\hline Deposits & 86.7 & 120.3 & 112.1 & 104.8 & 112.0 & 110.8 & 105.6 & 110.8 & 122.7 \\
\hline Demand deposits & 22.6 & 26.3 & 22.3 & 23.3 & 25.2 & 26.1 & 18.2 & 18.8 & 24.3 \\
\hline Public enterprises & 17.4 & 19.4 & 17.0 & 18.2 & 18.7 & 18.3 & 13.8 & 13.6 & 17.0 \\
\hline Private sector & 5.2 & 6.9 & 5.3 & 5.1 & 6.5 & 7.8 & 4.4 & 5.2 & 7.3 \\
\hline Time and savings deposits & 35.2 & 44.7 & 49.9 & 43.5 & 42.2 & 44.8 & 42.9 & 41.2 & 44.0 \\
\hline Foreign currency deposits & 28.9 & 49.3 & 40.0 & 38.0 & 44.6 & 39.8 & 44.5 & 50.9 & 54.4 \\
\hline Borrowing from BOM & 10.9 & 0.8 & 0.8 & 0.9 & 5.7 & 2.3 & 2.3 & 2.0 & 2.4 \\
\hline \multicolumn{10}{|l|}{ Memorandum items: } \\
\hline Net foreign assets (million U.S. dollars) & 71.6 & 88.3 & 43.4 & 32.5 & 36.6 & 29.0 & 30.0 & 34.0 & 33.7 \\
\hline Foreign currency deposits (million U.S. dollars) & 41.7 & 60.6 & 48.9 & 45.4 & 52.2 & 44.2 & 42.8 & 50.2 & 51.4 \\
\hline
\end{tabular}

Sources: Bank of Mongolia; and Fund staff estimates. 
Table 31. Mongolia: Net Credit to Government, 1996-99

(In billions of togrogs)

\begin{tabular}{|c|c|c|c|c|c|c|c|c|c|}
\hline & \multirow{2}{*}{$\begin{array}{l}1996 \\
\text { Dec. }\end{array}$} & \multirow{2}{*}{$\begin{array}{l}1997 \\
\text { Dec. }\end{array}$} & \multicolumn{4}{|c|}{1998} & \multicolumn{3}{|c|}{1999} \\
\hline & & & Mar. & June & Sep. & Dec. & Mar. & June & Sep. \\
\hline Net credit to government from the banking system & 20.5 & 21.3 & 29.1 & 33.4 & 41,0 & 47.9 & 58.1 & 68.7 & 44.0 \\
\hline Claims & 51.5 & 65.9 & 61.4 & 64.8 & 70.1 & 70.9 & 83.8 & 95.8 & 78.1 \\
\hline Minus: Deposits & 31.0 & 44.5 & 32.3 & 31.5 & 29.1 & 23.0 & 25.7 & 27.1 & 34.0 \\
\hline Bank of Mongolia (net) & 30.6 & 17.6 & 24.3 & 24.2 & 26.3 & 29.7 & 33.B & 41.7 & 29.5 \\
\hline Claims on Government & 39.0 & 30.4 & 29.9 & 29,9 & 32.0 & 32.6 & 40.9 & 46.9 & 35.9 \\
\hline Advance firancing to budget & 8.4 & 0.4 & 0.0 & 0.0 & 2.3 & 2.8 & 11.7 & 11.0 & 0.0 \\
\hline Louns to the MoF in CFC & 6.9 & 0.0 & 0.0 & 0.0 & 0.0 & 0.0 & 0.0 & 0.0 & 0.0 \\
\hline FX financing without togrog funds & 7.6 & 4.0 & 3.9 & 3.9 & 3.7 & 3.7 & 3.1 & 3.1 & 3.1 \\
\hline Govemment bonds & 15.5 & 13.8 & 13.8 & 13.8 & 19.6 & 19.6 & 19.6 & 19.6 & 19.6 \\
\hline Reconstruction bonds & 15.5 & 9.8 & 13.8 & 13.8 & 19.6 & 19.6 & 19.6 & 19.6 & 19.6 \\
\hline Treasury bills & 0.0 & 4.0 & 0.0 & 0.0 & 0.0 & 0.0 & 0.0 & 0.0 & 0.0 \\
\hline MARA account & 0.6 & 5.8 & 5.8 & 5.8 & 0.0 & 0.0 & 0.0 & 0.0 & 0.0 \\
\hline Treasury IMF account & 0.0 & 6.4 & 6.4 & 6.4 & 6.4 & 6.4 & 6.4 & 13.2 & 13.2 \\
\hline Less: Deposits & 8.4 & 12.8 & 5.7 & 5.8 & 5.8 & 2.9 & 7.0 & 5.2 & 6.3 \\
\hline Central Goverument & 7.9 & 12.3 & 4.4 & 5.2 & 5.4 & 2.3 & 6.9 & 5.1 & 6.3 \\
\hline Budget deposits & 4.2 & 9.7 & 2.5 & 2.9 & 3.4 & 0.5 & 4.7 & 1.5 & 0.7 \\
\hline In domestic currency & 0.4 & 2.9 & 1.6 & 2.3 & 0.3 & 0.1 & 3.4 & 1.4 & 0.6 \\
\hline In foreign currency & 3.8 & 6.8 & 1.0 & 0.5 & 3.1 & 0.4 & 1.3 & 0.1 & 0.1 \\
\hline Budget reserves & 3.7 & 2.6 & 1.8 & 2.3 & 2.0 & 1.8 & 2.2 & 3.6 & 5.5 \\
\hline Budget investrnent funds & 0.0 & 0.0 & 0.1 & 0.0 & 0.0 & 0,0 & 0.0 & 0.1 & 0.1 \\
\hline Local Government & 0.5 & 0.5 & 1.3 & 0.6 & 0.4 & 0.6 & 0.1 & 0.0 & 0.0 \\
\hline Budget revenue & 0.5 & 0.5 & 1.3 & 0.6 & 0.4 & 0.6 & 0.1 & 0.0 & 0.0 \\
\hline Comercial banks (net) & -10.1 & 3.7 & 4.9 & 9.2 & 14.7 & 18.2 & 24.3 & 27.0 & 14.5 \\
\hline Claims on Government & 12.5 & 35.5 & 31.5 & 34.9 & 38.1 & 38.3 & 43.0 & 48.9 & 42.2 \\
\hline Advance financing of MoF & 0.6 & 0.4 & 0.4 & 0.7 & 1.1 & 0.2 & 0.2 & 0.6 & 1.2 \\
\hline Government securities & 3.9 & 34.8 & 30.8 & 33.8 & 37.0 & 35.8 & 41,5 & 46.4 & 39.5 \\
\hline Inherited and directed credits & 7.7 & 0.0 & 0,0 & 0.0 & 0.0 & 0.0 & 0.0 & 0.0 & 0.0 \\
\hline Accrued interest receivable & 0.0 & 0.0 & 0.0 & 0.2 & 0.0 & 2.3 & 1.2 & 1.9 & 1.5 \\
\hline Projects & 0.0 & 0,0 & 0.0 & 0.0 & 0.0 & 0.0 & 0.0 & 0.0 & 0.0 \\
\hline Nontrade settlement & 0.0 & 0.0 & 0.0 & 0.0 & 0.0 & 0.0 & 0.0 & 0.0 & 0.0 \\
\hline Other & 0.4 & 0.3 & 0.3 & 0.3 & 0.0 & 0.0 & 0.0 & 0.0 & 0.0 \\
\hline FX financing without togrog funds & 0.4 & 0.3 & 0.3 & 0.3 & 0.0 & 0.0 & 0.0 & 0.0 & 0.0 \\
\hline Takeover of agricultural loans & 0.0 & 0.0 & 0.0 & 0,0 & 0.0 & 0.0 & 0.0 & 0.0 & 0.0 \\
\hline Less: Government deposits & 22.6 & 31.7 & 26.6 & 25.7 & 23.3 & 20.1 & 18.6 & 22.0 & 27.7 \\
\hline Central Government & 19.5 & 28.6 & 23.8 & 23.0 & 19.7 & 17.2 & 16.0 & 18.4 & 23.2 \\
\hline Budget deposits & 7.9 & 11.7 & 4.0 & 6.8 & 3.9 & 3.3 & 2.6 & 4.8 & 2.2 \\
\hline Budget reserves & 2.5 & 1.4 & 1.4 & 1.4 & 1.4 & 0.9 & 0.9 & 0.9 & 2.1 \\
\hline Budget funds with BITI & 0.4 & 0.4 & 0.4 & 0.1 & 0.2 & 0.0 & 0.0 & 0.1 & 0.0 \\
\hline Current account of budget units & 3.8 & 2.7 & 5.2 & 6.6 & 5.5 & 3.1 . & 4.6 & 5.8 & 8.5 \\
\hline Social security and peasion funds & 4.4 & 7.9 & 8.0 & 7.6 & 6.8 & 8.2 & 6.2 & 6.7 & 10.3 \\
\hline Privatization funds & 0.2 & 0.2 & 0.4 & 0.1 & 0.1 & 0.1 & 0.0 & 0.0 & 0.0 \\
\hline Budget investment funds & 0.3 & 0.0 & 0.2 & 0.3 & 0.2 & 0.0 & 0.0 & 0.0 & 0.0 \\
\hline Time deposits & 0.0 & 4.3 & 4.4 & 0.2 & 1.7 & 1.7 & 1.7 & 0.2 & 0.1 \\
\hline Repayable assistance to Reconstruction Bank & 0.0 & 0.0 & 0.0 & 0.0 & 0.0 & 0.0 & 0.0 & 0.0 & 0.0 \\
\hline Local Govermment & 2.3 & 2.4 & 2.3 & 2.4 & 2.9 & 2.0 & 2.2 & 3.0 & 3.7 \\
\hline Budget revenue & 0.0 & 1.1 & 0.7 & 1.1 & 1.3 & 0.5 & 0.7 & 1.4 & 1.8 \\
\hline Reserves & 0.0 & 0.0 & 0.0 & 0.0 & 0.0 & 0.0 & 0.0 & 0.0 & 0.0 \\
\hline Current account of budget units & 0.0 & 1.3 & 1.3 & 1.1 & 1.4 & 1.1 & 1.1 & 1.3 & 1.6 \\
\hline Budget investment funds & 0.0 & 0.0 & 0.2 & 0.2 & 0.2 & 0.3 & 0.3 & 0.3 & 0.3 \\
\hline Time deposits & 0.5 & 0.1 & 0.0 & 0.0 & 0.0 & 0.0 & 0.0 & 0.0 & 0.0 \\
\hline Accounts outside budget & 0.2 & 0.6 & 0.5 & 0.2 & 0.8 & 0.9 & 0.4 & 0.5 & 0.7 \\
\hline
\end{tabular}

Sources: Mongolian authorities; and Fund staff estimates. 
Table 32. Mongolia: Interest Rates, 1996-99

(In percent per annum)

\begin{tabular}{|c|c|c|c|c|c|c|c|c|c|}
\hline & \multirow{2}{*}{$\begin{array}{l}1996 \\
\text { Dec. }\end{array}$} & \multirow{2}{*}{$\begin{array}{l}1997 \\
\text { Dec. }\end{array}$} & \multicolumn{4}{|c|}{1998} & \multicolumn{3}{|c|}{1999} \\
\hline & & & Mar. & June & Sep. & $\overline{\text { Dec. }}$ & Mar. & June & Sep. \\
\hline \multicolumn{10}{|l|}{ Deposit rates } \\
\hline Current accounts & 2 & 2 & $0.8-9$ & $3-9$ & $0.8-6$ & $0.8-6$ & $0.8-6$ & $0.8-6$ & $3-6$ \\
\hline Savings deposit accounts & $12-35$ & $4-35$ & 4-27 & $4-20$ & $4-20$ & $4-20$ & $4-20$ & $4-20$ & 4-20 \\
\hline \multicolumn{10}{|l|}{ Time deposit accounts } \\
\hline $0-1$ year & $13-60$ & $6-70$ & $12-43$ & $12-43$ & $6-39$ & $6-43$ & $6-43$ & $6-43$ & $5-43$ \\
\hline $1-3$ year & 27 & $36-43$ & 24 & $18-24$ & $12-30$ & $24-30$ & $24-30$ & $24-30$ & $24-30$ \\
\hline \multicolumn{10}{|l|}{ Foreign currency } \\
\hline $1-3$ year & $4-43$ & $4-43$ & $2-43$ & 1.27 & $1-24$ & $1-24$ & $1-24$ & $1-24$ & $2-24$ \\
\hline \multicolumn{10}{|l|}{ Loan rates } \\
\hline Bank of Mongolia & $72-109$ & $45-50$ & 27.5 & 37.5 & 27.5 & 23.3 & 23.3 & 17.5 & 17.5 \\
\hline \multicolumn{10}{|c|}{$\begin{array}{l}\text { Commercial loans } 1 / \\
\text { Short-term (less than } 1 \text { year) }\end{array}$} \\
\hline Domestic currency & $\cdots$ & $\cdots$ & 47.6 & 47.8 & 47.4 & 45.8 & 47.2 & 42.2 & 42.4 \\
\hline Foreign currency & $\cdots$ & $\ldots$ & 42.1 & 41.9 & 42.9 & 34.2 & 37.2 & 34.1 & 30.6 \\
\hline
\end{tabular}

Source: Bank of Mongolia.

1/ Average rates. 
Table 33. Mongolia: Selected Financial Indicators of Commercial Banks, 1998-99

\begin{tabular}{|c|c|c|c|c|}
\hline \multirow{2}{*}{ Bank } & \multicolumn{2}{|c|}{$\begin{array}{c}\text { Total Assets } \\
\text { (In billions of togrogs) }\end{array}$} & \multicolumn{2}{|c|}{$\begin{array}{l}\text { Gross Nonperforming Loans } \\
\text { (Percent of total loans) }\end{array}$} \\
\hline & & & Aug. 1998 & Sep. 1999 \\
\hline Trade and Development Bank & 78.6 & 5.6 & 14.2 & 45.6 \\
\hline Golornt Bank & 22.6 & 2.3 & 10.8 & 9.8 \\
\hline Agriculture Bank & 9.2 & -6.0 & 65.2 & 77.1 \\
\hline ITI Bank & 11.6 & -12.6 & 55.3 & 92.1 \\
\hline Savings Bank & 35.3 & 0.9 & 3.7 & 28.9 \\
\hline Post Bank & 6.8 & 1.2 & 55.9 & 19.1 \\
\hline Reconstruction Bank & 9.2 & -5.1 & 84.5 & 100.0 \\
\hline Exim Bank & 2.6 & 0.2 & 57.4 & 93.5 \\
\hline Erel Bank & 2.5 & 1.7 & 0.0 & 0.0 \\
\hline Bayanbogd Bank 1/ & 0.7 & 0.4 & 2.4 & 6.6 \\
\hline Innovation Bank & 2.8 & 0.7 & 82.9 & 45.4 \\
\hline Asian Investment Bank 1/ & 0.9 & 0.4 & 0.0 & 18.1 \\
\hline Transport Bank & 2.4 & 1.0 & 5.6 & 10.9 \\
\hline Mercury Bank 1/ & 0.5 & 0.0 & 100.0 & 97.6 \\
\hline Business Bank 1/ & 2.7 & 0.5 & 74.0 & 37.0 \\
\hline MM Invest Bank 1/ & 0.9 & 0.8 & 0.0 & 0.0 \\
\hline Credit Bank & 1.7 & 1.2 & 0.0 & 6.8 \\
\hline Ulaarbaatar Bank 1/ & 2.9 & 0.6 & 0.0 & 0.6 \\
\hline Zoos Bank & 1.1 & 1.0 & $\ldots$ & 0.0 \\
\hline Anod Bank & 3.0 & 1.0 & $\ldots$ & 0.0 \\
\hline
\end{tabular}

Sources: Bank of Mongolia; and Fund staff estimates.

1/ The licenses of these banks have been withdrawn by the Bank of Mongolia. 
Table 34. Mongolia: Balance of Payments, 1994-98

(In millions of U.S. dollars)

\begin{tabular}{|c|c|c|c|c|c|}
\hline & 1994 & 1995 & 1996 & 1997 & 1998 \\
\hline Trade baiance & -3.5 & -3.3 & -87.4 & 30.2 & -120.1 \\
\hline Exports, f.o.b. & 367.0 & 485.6 & 423.4 & 568.5 & 462.3 \\
\hline Copper & 188.7 & 257.6 & 174.5 & 199.1 & 124.8 \\
\hline Noncopper & 178.3 & 228.0 & 248.8 & 369.4 & 337.5 \\
\hline Imports, c.i.f. & -370.5 & .488 .9 & -510.8 & -538.3 & -582.4 \\
\hline Services balance & -14.2 & -23.4 & -6.0 & -7.3 & -11.5 \\
\hline Recelpts & 39.9 & 42.6 & 55.7 & 80.0 & 77.9 \\
\hline Payments & -54.1 & -66.0 & -61.7 & -87.3 & -89.4 \\
\hline Income, net & -19.3 & -22.7 & -13.3 & -14.3 & 0.4 \\
\hline Official interest payments & -14.3 & -9.4 & -9.3 & -7.6 & -9.2 \\
\hline Private unrequited transfers & -3.0 & -2.8 & 6.2 & 4.2 & 2.5 \\
\hline \multicolumn{6}{|l|}{ Current account deficit $(-)$, excluding } \\
\hline official transfers & -40.3 & -52.2 & -100.5 & 12.8 & -128.8 \\
\hline Public unrequited transfers (net) & 77.6 & 79.1 & 63.6 & 61.5 & 53.3 \\
\hline Official grants & 69.5 & 70.9 & 56.2 & 54.8 & 45.8 \\
\hline Other official transfers (net) & 8.1 & 8.2 & 7.4 & 6.7 & 7.5 \\
\hline Financial and Capital account & -36.2 & 19.0 & 41.2 & 52.5 & 116.8 \\
\hline Direct investment & 7.0 & 9.8 & 15.9 & 20.0 & 18.9 \\
\hline Medium- and long-term loans (net) & 7.9 & 50.9 & 56.0 & 109.8 & 93.4 \\
\hline Disbursements & 60.3 & 96.2 & 93.4 & 142.3 & 122.5 \\
\hline Amortizations & $-\$ 2.4$ & -45.3 & -37.3 & -32.5 & -29.1 \\
\hline Commercial Banks, (net) & 6.2 & -8.1 & -9.3 & -18.1 & 40.0 \\
\hline Short-term & -57.2 & -33.6 & -21.5 & -59.2 & -35.5 \\
\hline Enrors and omissions & -10.9 & -54.5 & -30.4 & .69 .6 & -69.8 \\
\hline Overall balance & -9.5 & -8.7 & -26.1 & 57.2 & -28.5 \\
\hline Financing & 9.5 & 8.7 & 26.1 & -57.2 & 28.5 \\
\hline Change in net official reserves (increase -) & -10.0 & 5.1 & 20.1 & -64.3 & 15.0 \\
\hline Anears accumulated (+) or payments (-) & -37.0 & -2.4 & 5.9 & -1.6 & 13.5 \\
\hline Exceptional financing (rescheduling) & 56.5 & 5.9 & 0.0 & 8.7 & 0.0 \\
\hline \multicolumn{6}{|l|}{ Memorandum items: } \\
\hline $\begin{array}{l}\text { Current account deficit (-), excluding } \\
\text { official transfers (in percent of GDP) }\end{array}$ & -5.8 & -5.5 & -10.0 & 1.3 & -126 \\
\hline $\begin{array}{l}\text { official transters (in percent of GDP) } \\
\text { Net official reserves (end of period) }\end{array}$ & 37.2 & 67.9 & 54.0 & $\begin{array}{r}1.3 \\
89.6\end{array}$ & $\begin{array}{r}-12.6 \\
74.9\end{array}$ \\
\hline (ln weeks of total imports) & 5.2 & 7.2 & $\$ .5$ & 8.7 & 6.7 \\
\hline Gross official reserves (end of period) & 92.4 & 114.9 & 97.8 & 137.2 & 123.2 \\
\hline (In weeks of tota! imports) & 13.0 & 12.2 & 10.0 & 13.3 & 11.0 \\
\hline Outstanding arrears (end of period) & 3.8 & 1.4 & 7.3 & 0.9 & 13.5 \\
\hline
\end{tabular}

Sources: Mongolian authorities; and Fund staff estimates. 
Table 35. Mongolia: Principal Exports, 1994-98

(In millions of U.S. dollars, unit value in U.S. dollars)

\begin{tabular}{|c|c|c|c|c|c|}
\hline & 1994 & 1995 & 1996 & 1997 & 1998 \\
\hline Copper concentrate (value) & 188.7 & 257.6 & 174.5 & 199.1 & 124.8 \\
\hline Volume (concentrate in ' 000 tous) & 405.4 & 415.6 & 429.2 & 479.7 & 485.7 \\
\hline Refined copper equivalent & 119.2 & 116.0 & 124.6 & 119.1 & 80.5 \\
\hline Unit value-refined (per ton) & $1,583.1$ & $2,219.4$ & $1,400.9$ & $1,671.7$ & $1,550.0$ \\
\hline Molybdenum concentrate & 17.0 & 15.9 & 10.8 & 15.7 & 12.1 \\
\hline Volume (tons) & $5,247.1$ & $3,800,0$ & $4,787.0$ & $4,109.3$ & $4,131.3$ \\
\hline Unit value (per ton) & $3,239.9$ & $4,184,2$ & $2,256.1$ & $3,820.6$ & $2,919.2$ \\
\hline Fluorite ore & 1.6 & 0.6 & $\ldots$ & $\cdots$ & 0.0 \\
\hline Volume ('000 tons) & 16.2 & 5.3 & $\ldots$ & $\ldots$ & 0.0 \\
\hline Unit value (per ton) & 98.8 & 113.2 & $\cdots$ & $\cdots$ & $\cdots$ \\
\hline Fluorite concentrate & 11.0 & 18.4 & 22.6 & 23.4 & 18.5 \\
\hline Valume ('000 tons) & 88.0 & 120.4 & 155.2 & 178.0 & 156.8 \\
\hline Unit value (per kg) & 125.0 & 152.8 & 145.6 & 131.5 & 117.6 \\
\hline Gold & 11.4 & 51.9 & 52.4 & 117.0 & 117.2 \\
\hline Volume (kg) & $1,104,1$ & $4,215.6$ & $4,257.7$ & $11,695.9$ & $12,544.1$ \\
\hline Unit value (per kg) & $10,325.2$ & $12,310.3$ & $12,310.3$ & $10,003.5$ & $9,339.9$ \\
\hline Sawn wood & 7.8 & 4.4 & 9.1 & 20.8 & 34.1 \\
\hline Volume ('000 cubic meters) & 78.7 & 44.8 & 96.4 & 168.7 & 268.1 \\
\hline Unit value (per cubic meter) & 99.1 & 98.2 & 94.4 & 123.3 & 127.1 \\
\hline Cashmere tops & 6.8 & 3.8 & 2.2 & 0.9 & 0.5 \\
\hline Volume (tons) & 71.6 & 34.0 & 28.3 & 12.5 & 9.6 \\
\hline Unit value (per kg) & 95.0 & 112.7 & 77.7 & 72.0 & 48.4 \\
\hline Cashmere, dehaired & 26.1 & 45.2 & 49,4 & 31.6 & 33.0 \\
\hline Volume (tons) & 341.0 & 507.3 & 686.9 & 590.4 & 849.8 \\
\hline Unit value (per kg) & 76.5 & 89.1 & 71.9 & 53.5 & 38.8 \\
\hline Cashmere garments & 3.6 & 7.8 & $\ldots$ & 10,4 & 5.0 \\
\hline Volume ("000 pieces) & 99.0 & 215.8 & $\ldots$ & 275.0 & 152.2 \\
\hline Unit value (per piece) & 36.4 & 36.2 & $\ldots$ & 37.8 & 32.8 \\
\hline Cashmere, greasy/raw & 3.5 & 0.4 & 8.5 & 16.2 & 0.3 \\
\hline Volume (tons) & 240.8 & 78.8 & 393.4 & 824.6 & 16.2 \\
\hline Unit valute (per kg) & 14.5 & 5.1 & 21.6 & 19.6 & 18.9 \\
\hline Textiles & $\ldots$ & $\ldots$ & $\ldots$ & 26.1 & 23.7 \\
\hline Volume ( 000 pieces) & $\ldots$ & $\cdots$ & $\ldots$ & $2,890.0$ & $3,786.1$ \\
\hline Unit price & $\cdots$ & $\ldots$ & $\ldots$ & 9.0 & 6.3 \\
\hline Camel wool, raw & 5.6 & 2.3 & 2.1 & 2.1 & 1.4 \\
\hline Volume (tons) & $2,505,4$ & 940.3 & $1,025.0$ & 904.2 & 664,3 \\
\hline Unit value (per kg) & 2.2 & 2.4 & 2.0 & 2.3 & 2.1 \\
\hline Skin and hides & 18.1 & 21.8 & 19.1 & 21.1 & 23.8 \\
\hline Volume ('000 pieces) & $2,891.9$ & $2,763.6$ & $2,300.0$ & $2,600.0$ & $2,798.5$ \\
\hline Unil price & 6.3 & 7.9 & 8.3 & 8.1 & 8.5 \\
\hline Leather goods & 1.3 & 0.1 & 0.1 & 3.7 & 0.3 \\
\hline Volume (000 pieces) & 17.7 & 1.2 & $\ldots$ & 37.0 & 2.1 \\
\hline Unit value (per piece) & 73.5 & 83.3 & ... & 100.0 & 149.8 \\
\hline Sheepsikin coats & 0.9 & 0.2 & 0.03 & 0.0 & 0.1 \\
\hline Volume ( 000 pieces) & 11.8 & 3.7 & 0.5 & 0.4 & 0.8 \\
\hline Unit value (per píece) & 76.3 & 54.1 & 60.0 & 56.5 & 158.0 \\
\hline Carpets & 0.9 & 0.2 & 0.1 & 0.0 & 0.0 \\
\hline Volume (c000 meters) & 68.6 & 11.5 & 2.2 & 3.1 & 0.0 \\
\hline Unit value (per meter) & 13.1 & 17.4 & 45.5 & 14.5 & $\ldots$ \\
\hline Meat & 6.3 & 2.8 & 4.7 & 9.0 & 8.7 \\
\hline Volume (000 tons) & 5.1 & 2.1 & 3.3 & 6.4 & 6.7 \\
\hline Unit valite (per ton) & $1,237.6$ & $1,333.3$ & $1,424.2$ & $1,406.3$ & $3,287.6$ \\
\hline Scrap Metal & 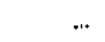 & $\cdots$ & $\cdots$ & 16.1 & 9.5 \\
\hline Voilume ('000 tons) & $\ldots$ & $\ldots$ & $\ldots$ & 90.1 & 32,2 \\
\hline Unit vahue (per ton) & $\cdots$ & $\cdots$ & $\cdots$ & 179.1 & 294.6 \\
\hline Other & 56.4 & 52.2 & 67.7 & 55.2 & 49.6 \\
\hline Total & 367.0 & 485.6 & 423.4 & 568.5 & 462.3 \\
\hline
\end{tabular}

Sources: Mongolian authorities; ard Fund staff estimates. 
Table 36. Mongolia: Merchandise Imports, 1994-98 1/

(In millions of U.S. dollars)

\begin{tabular}{lccccc}
\hline & 1994 & 1995 & 1996 & 1997 & 1998 \\
\hline Food & 20.4 & 34.3 & 62.2 & 76.5 & 80.9 \\
Energy & 75.4 & 67.7 & 87.8 & 91.2 & 91.3 \\
Equipment goods & 92.7 & 137.9 & 173.6 & 170.8 & 194.9 \\
Raw materials and spare parts & 31.7 & 71.1 & 67.2 & 65.4 & 62.7 \\
Consumer goods & 38.2 & 65.8 & 47.1 & 64.2 & 73.5 \\
$\begin{array}{l}\text { Other imports } \\
\quad 112.1\end{array}$ & 112.1 & 72.9 & 70.2 & 79.1 \\
$\quad$ Total & 370.5 & 488.9 & 510.8 & 538.3 & 582.4 \\
$\begin{array}{l}\text { Memorandum items: } \\
\quad \text { Nonfood imports } \\
\text { Nonenergy imports }\end{array}$ & & & & & \\
\hline
\end{tabular}

Sources: Mongolian authorities; and Fund staff estimates.

1/ On a c.i.f. basis. 
Table 37. Mongolia: Direction of Trade, 1994-98 1/

\begin{tabular}{|c|c|c|c|c|c|}
\hline & 1994 & 1995 & 1996 & 1997 & 1998 \\
\hline Exports & 100.0 & 100.0 & 100.0 & 100.0 & 100.0 \\
\hline Countries of the former CMEA & 41.0 & 32.3 & 25.1 & 10.6 & 11.9 \\
\hline Former U.S.S.R. & 40.6 & 30.3 & 24.1 & 10.6 & 11.9 \\
\hline Russia & $\ldots$ & 14.5 & 20.6 & 10.4 & 11.8 \\
\hline Kazakhstan & $\ldots$ & 15.7 & 3.4 & 0.2 & 0.1 \\
\hline Other & $\ldots$ & 0.1 & 0.1 & 0.1 & 0.0 \\
\hline Former Czechoslovakia 2/ & 0.3 & 2.0 & 1.0 & 0.0 & 0.0 \\
\hline Korea, Democratic People's Republic of & 0.1 & 0.0 & 0.0 & 0.0 & 0.0 \\
\hline EU countries & 6.2 & 12.6 & 9.8 & 10.4 & 9.5 \\
\hline Belgium & 0.5 & 0.5 & 0.5 & 0.3 & 1.0 \\
\hline France & 0.1 & 0.1 & 0.1 & 0.1 & 0.1 \\
\hline Germany & 0.6 & 1.6 & 1.1 & 1.4 & 0.6 \\
\hline Italy & 2.7 & 2.4 & 2.5 & 2.5 & 2.9 \\
\hline Netherlands & 0.9 & 1.0 & 1.0 & 0.6 & 1.0 \\
\hline United Kingdom & 1.4 & 3.9 & 4.4 & 5.3 & 3.7 \\
\hline Other & 0.0 & 3.1 & 0.2 & 0.2 & 0.2 \\
\hline Other & 50.0 & 55.1 & 65.1 & 79.1 & 78.5 \\
\hline China & 19.4 & 16.4 & 17.7 & 21.8 & 29.3 \\
\hline Austria & 0.0 & 0.0 & 0.0 & 0.0 & 0.0 \\
\hline Finland & 0.6 & 0.4 & 0.0 & 0.0 & 1.1 \\
\hline Hong Kong, China & 0.7 & 0.6 & 0.6 & 1.1 & 2.6 \\
\hline Japan & 13.1 & 10.0 & 8.5 & 8.4 & 3.7 \\
\hline Korea & 5.9 & 5.5 & 8.0 & 9.8 & 9.6 \\
\hline Singapare & 0.0 & 0.4 & 0.0 & 0.0 & 0.4 \\
\hline Switzerland & 6.4 & 16.2 & 25.4 & 31.0 & 20.4 \\
\hline United States & 3.7 & 5.5 & 4.4 & 5.6 & 8.5 \\
\hline \multicolumn{6}{|l|}{ Federal Republic of Yugoslavia } \\
\hline (Serbja/Montenegro) & 0.2 & 0.0 & 0.1 & 0.0 & 0.0 \\
\hline Other & 2.8 & 0.1 & 0.4 & 1.4 & 2.9 \\
\hline Imports & 100.0 & 100.0 & 100.0 & 100.0 & 100.0 \\
\hline Countries of the former CMEA. & 60.1 & 53.7 & 41.1 & 37.3 & 35.4 \\
\hline Former U.S.S.R. & 59.2 & 50.7 & 37.6 & 36.3 & 31.5 \\
\hline Russia & $\ldots$ & 50.1 & 34.2 & 35.4 & 29.9 \\
\hline Kazakhstan & $\ldots$ & 0.2 & 0.2 & 0.4 & 0.7 \\
\hline Other & $\ldots$ & 0.4 & 3.2 & 0.5 & 1.0 \\
\hline Bulgaria & 0.1 & 0.1 & 0.4 & 0.7 & 0.8 \\
\hline Former Czechoslovakia 2/ & 0.3 & 2.4 & 1.8 & 1.6 & 1.3 \\
\hline Hungary & 0.1 & 0.1 & 0.5 & 0.4 & 0.4 \\
\hline Poland & 0.4 & 0.3 & 0.8 & 1.1 & 1.3 \\
\hline Korea, Democratic People's Republic of & 0.0 & 0.1 & 0.0 & 0.0 & 0.0 \\
\hline EU countries & 5.3 & 7.8 & 13.5 & 19.9 & 13.4 \\
\hline Denmark & 0.6 & 1.0 & 0.5 & 2.8 & 0.2 \\
\hline France & 0.2 & 0.4 & 0.3 & 2.3 & 5.3 \\
\hline Germany & 4.0 & 4.4 & 4.7 & 4.3 & 5.1 \\
\hline United Kingdom & 0.5 & 0.7 & 0.7 & 1.1 & 0.7 \\
\hline Other & 0.0 & 1.3 & 7.3 & 9.3 & 2.1 \\
\hline Other & 33.6 & 38.5 & 45.4 & 42.8 & 51.2 \\
\hline China & 9.6 & 10.7 & 14.6 & 12.9 & 11.6 \\
\hline Austria & 0.0 & 1.3 & 0.1 & 0.0 & 3.2 \\
\hline Finland & 0.1 & 0.3 & 0.2 & 0.4 & 1.2 \\
\hline Hong Kong, China & 4.3 & 1.5 & 0.9 & 1.2 & 1.6 \\
\hline India & 0.0 & 0.3 & 0.1 & 0.1 & 0.3 \\
\hline Japan & 6.2 & 11.0 & 17.5 & 7.4 & 11.8 \\
\hline Korea & 4.3 & 5.2 & 4.0 & 4.4 & 7.5 \\
\hline Singapore & 1.4 & 1.8 & 2.9 & 3.8 & 3.4 \\
\hline Switzerland & 1.5 & 1.5 & 1.0 & 0.4 & 0.2 \\
\hline United States & 5.0 & 3.5 & 2.5 & 7.9 & 7.2 \\
\hline \multicolumn{6}{|l|}{ Federal Republic of Yugosiavia } \\
\hline (Serbia/Montenegro) & 0.0 & 0.0 & 0.0 & 0.0 & 0.0 \\
\hline Other & 1.1 & 1.4 & 1.6 & 4.4 & 3.1 \\
\hline
\end{tabular}

Sources: Mongolian authorities; and Fund staff estimates.

V/ Data for exports of copper refer to country of initial purchaser rather than country of destination of final product.

2/ Data after 1993 refer to Czech Republic and Slovak Republic. 
Table 38. Mongolia: Services and Income Accounts, 1996-98 1/

(In millions of U.S. dollars)

\begin{tabular}{|c|c|c|c|c|c|c|}
\hline & \multicolumn{2}{|c|}{1996} & \multicolumn{2}{|c|}{1997} & \multicolumn{2}{|c|}{1998} \\
\hline & Receipts & Payments & Receipts & Payments & Receipts & Payments \\
\hline \multicolumn{7}{|l|}{ Services } \\
\hline Passenger fares & 9.7 & 18.0 & 12.3 & 5.0 & 11.8 & 4.6 \\
\hline Railway & 5.2 & 8.6 & 6.3 & 2.7 & 6.0 & 2.1 \\
\hline Airline & 4.5 & 9.4 & 6.0 & 2.3 & 5.8 & 2.5 \\
\hline Port services & 14.0 & 5.9 & 12.8 & 11.0 & 18.2 & 15.9 \\
\hline Transit fees & 4.0 & 0.0 & 4.1 & 6.0 & 2.2 & 2.6 \\
\hline Travel $2 /$ & 10.0 & 19.4 & 39.8 & 45.3 & 35.0 & 45.1 \\
\hline Embassy expenses abroad & 2.6 & 4.1 & 1.9 & 4.4 & 2.7 & 4.5 \\
\hline Embassies/international organizations & 10.0 & 0.0 & 0.6 & 0.2 & 0.5 & 0.0 \\
\hline Commissions & 0.0 & 0.3 & 0.0 & 0.7 & 0.0 & 0.8 \\
\hline Communications & 4.8 & 3.0 & 6.8 & 4.7 & 7.2 & 6.0 \\
\hline Technical Assistance & 0.0 & 9.0 & 0.0 & 9.0 & 0.0 & 6.5 \\
\hline Other & 0.6 & 2.0 & 1.7 & 1.0 & 0.3 & 3.4 \\
\hline Total & 55.7 & 61.7 & 80.0 & 87.3 & 77.9 & 89.4 \\
\hline \multicolumn{7}{|l|}{ Income } \\
\hline Direct investment income & 0.0 & 16.1 & 0.0 & 11.6 & 0.0 & 0.5 \\
\hline Interest on bank deposits & 13.4 & 1.3 & 6.1 & 1.1 & 10.1 & 0.0 \\
\hline Interest on debt & 0.0 & 9.3 & 0.0 & 7.6 & 0.0 & 9.2 \\
\hline Medium- and long-term & 0.0 & 9.3 & 0.0 & 7.6 & 0.0 & 8.7 \\
\hline Short-term & 0.0 & 0.0 & 0.0 & 0.0 & 0.0 & 0.5 \\
\hline Total & 13.4 & 26.7 & 6.1 & 20.3 & 10.1 & 9.7 \\
\hline
\end{tabular}

Sources: Mongolian authorities; and Fund staff estimates.

1/ A significant broadening in the coverage of the service sector was achieved in 1996.

2/ From 1997, estimated from data on arrivals and departures at border points. 
Table 39. Mongolia: Medium- and Long-Term Loan Disbursements, 1994-98

(In millions of U.S. collars)

\begin{tabular}{|c|c|c|c|c|c|}
\hline & 1994 & 1995 & 1996 & 1997 & 1998 \\
\hline Official loans & 60.3 & 90.7 & 84.4 & 134.3 & 94.4 \\
\hline Japan & 2.4 & 22.2 & 29.6 & 12.9 & 36.4 \\
\hline Balance of payments support & 0.0 & 0.0 & 0.0 & 0.0 & 0.0 \\
\hline Project & 2.4 & 22.2 & 29.6 & 12.9 & 36.4 \\
\hline Germany & 7.3 & 2.5 & 3.3 & 5.1 & 4.7 \\
\hline China & 0.0 & 0.0 & 4.4 & 7.1 & 0.3 \\
\hline Balance of payments support & 0.0 & 0.0 & 0.0 & 0.0 & 0.0 \\
\hline Project & 0.0 & 0.0 & 4.4 & 7.1 & 0.3 \\
\hline Korea & 0.8 & 4.4 & 0.0 & 0.0 & 0.1 \\
\hline Russia & 9.1 & 1.5 & 0.0 & 0.0 & 0.0 \\
\hline India & 1.7 & 1.5 & 0.0 & 0.0 & 0.0 \\
\hline Norway & 0.0 & 0.0 & 2.0 & 7.0 & 2.7 \\
\hline Kuwait & 0.0 & 0.0 & 0.0 & 0.7 & 1.7 \\
\hline Worid Bank & 19.0 & 14.0 & 11.0 & 34.0 & 16.4 \\
\hline Balance of payments support & 17.5 & 9.7 & 1.8 & 9.3 & 0.0 \\
\hline Project & 0.0 & 3.6 & 8.8 & 24.1 & 2.4 \\
\hline Technical assistance & 1.5 & 0.7 & 0.4 & 0.6 & 14.0 \\
\hline Asian Development Bank & 20.0 & 44.6 & 34.1 & 67.4 & 31.8 \\
\hline Balance of payments support & 10.3 & 32.7 & 0.0 & 35.9 & 0.0 \\
\hline Project & 9.7 & 11.9 & 34.1 & 31.5 & 5.1 \\
\hline Technical assistance & 0.0 & 0.0 & 0.0 & 0.0 & 26.8 \\
\hline IFAD & 0.0 & 0.0 & 0.0 & 0.3 & 0.3 \\
\hline Commercial loans & 0.0 & 5.5 & 9.0 & 8.0 & 30.3 \\
\hline Copper mine & 0.0 & 0.0 & 0.0 & 0.0 & 0.0 \\
\hline Mini-metal project & 0.0 & 0.0 & 0.0 & 0.0 & 0.0 \\
\hline Airplanes & 0.0 & 0.0 & 0.0 & 0.0 & 28.8 \\
\hline Leather factory & 0.0 & 5.5 & 9.0 & 6.0 & 0.0 \\
\hline Commercial bank & 0.0 & 0.0 & 0.0 & 2.0 & 1.5 \\
\hline Total & 60.3 & 96.2 & 93.4 & 142.3 & 124.7 \\
\hline
\end{tabular}

Sources: Mongolian authorities; and Fund staff estimates. 
Table 40. Mongolia: Short-Term Capital Flows, 1995-98

(In millions of U.S. dollars)

\begin{tabular}{|c|c|c|c|c|}
\hline & 1995 & 1996 & 1997 & 1998 \\
\hline Import trade credits & 0.0 & 6.1 & -6.1 & 0.0 \\
\hline Austria & 0.0 & 0.0 & 0.0 & 0.0 \\
\hline Japan & 0.0 & 0.0 & 0.0 & 0.0 \\
\hline France & 0.0 & 0.0 & 0.0 & 0.0 \\
\hline United States & 0.0 & 0.0 & 0.0 & 0.0 \\
\hline Other & 0.0 & 6.1 & -6.1 & 0.0 \\
\hline \multicolumn{5}{|l|}{$\begin{array}{l}\text { Amortization of import trade credits } \\
\text { (International Bank for Economic }\end{array}$} \\
\hline Cooperation balances) & 0.0 & 0.0 & 0.0 & 0.0 \\
\hline Prepayment for imports & 6.9 & 0.0 & -10.0 & 0.2 \\
\hline Japanese grant & 4.7 & 0.0 & -10.0 & 0.2 \\
\hline U.S. grant & 2.2 & 0.0 & 0.0 & 0.0 \\
\hline Export trade credits & -8.9 & 3.8 & -11.1 & -1.8 \\
\hline Copper receipts & -8.9 & 3.8 & -11.1 & -1.8 \\
\hline Counterpart of barter trade balance & 0.0 & 0.0 & 0.0 & 0.0 \\
\hline \multicolumn{5}{|l|}{ Position of non-bank sector vis a vis } \\
\hline BIS commercial banks, net & 0.0 & -1.0 & -1.0 & 0.0 \\
\hline Other & -31.6 & -30.4 & -31.0 & -33.9 \\
\hline Total & -33.6 & -21.5 & -59.2 & -35.5 \\
\hline
\end{tabular}

Sources: Mongolian authorities; and Fund staff estimates. 
Table 41. Mongolia: Official International Reserves of the Bank of Mongolia, 1995-98

(In millions of U.S. dollars, end of period)

\begin{tabular}{|c|c|c|c|c|}
\hline & 1995 & 1996 & 1997 & 1998 \\
\hline Gross foreign assets & 114.9 & 97.8 & 139.9 & 126.6 \\
\hline Cash & 0.2 & 4.9 & 1.5 & 2.9 \\
\hline SDR holdings & 2.6 & 0.4 & 0.7 & 0.5 \\
\hline Monetary gold & 34.5 & 53.6 & 24.6 & 9.1 \\
\hline Current account & 4.5 & 5.5 & 0.6 & 1.1 \\
\hline Deposit account & 70.9 & 33.4 & 78.7 & 89.6 \\
\hline Foreign government bills & 0.0 & 0.0 & 33.8 & 23.5 \\
\hline Foreign grants & 2.3 & 0.0 & 0.0 & 0.0 \\
\hline Short-term foreign liabilities & 47.0 & 43.8 & 50.3 & 51.7 \\
\hline Borrowing from banks & 0.0 & 0.0 & 0.0 & 0.0 \\
\hline \multicolumn{5}{|l|}{ International Bank for } \\
\hline Economic Cooperation & 0.0 & 0.0 & 0.0 & 0.0 \\
\hline International Investment Bank & 0.0 & 0.0 & 0.0 & 0.0 \\
\hline Use of Fund credit & 47.0 & 43.8 & 47.9 & 48.3 \\
\hline \multicolumn{5}{|l|}{ Foreign Currency Reserve Balances } \\
\hline of domestic commercial banks & 0.0 & 0.0 & 2.4 & 3.4 \\
\hline Net international reserves & 67.9 & 54.0 & 89.6 & 74.9 \\
\hline
\end{tabular}

Sources: Bank of Mongolia; and Fund staff estimates. 
Table 42. Mongolia: Selected Indicators of Commercial Bank Foreign Exchange Operations

\begin{tabular}{|c|c|c|c|c|c|c|c|}
\hline & \multirow[t]{2}{*}{1995} & \multirow[t]{2}{*}{1996} & \multirow[t]{2}{*}{1997} & \multirow[t]{2}{*}{1998} & \multicolumn{3}{|c|}{1999} \\
\hline & & & & & Mar. & June & Sep. \\
\hline Foreign assets (US\$ million) & 53.4 & 62.4 & 81.7 & 29.0 & 29.1 & 32.8 & 32.5 \\
\hline Cash & 7.9 & 22.6 & 5.6 & 3.1 & 4.0 & 3.3 & 6.6 \\
\hline Demand deposits & 41.5 & 18.3 & 44.4 & 19.0 & 13.6 & 16.3 & 2.5 \\
\hline Time deposits & 4.0 & 21.5 & 31.7 & 6.8 & 11.5 & 13.2 & 23.4 \\
\hline Foreign liabilities (US\$ million) & 4.4 & 12.4 & 15.1 & 22.1 & 21.5 & 22.1 & 16.9 \\
\hline Deposits of foreign banks and non-residents & 3.0 & 9.4 & 8.6 & 0.0 & 0.9 & 1.2 & 1.2 \\
\hline Loans from foreign banks & 1.4 & 3.0 & 6.4 & 22.1 & 20.6 & 20.9 & 15.7 \\
\hline Foreign currency denominated loans (USS million) & 18.8 & 15.3 & 20.2 & 44.3 & 41.6 & 44.7 & 41.9 \\
\hline \multicolumn{8}{|l|}{ Summary indicators (in percent) } \\
\hline FC loans/total loans & 13.6 & 14.2 & 31.9 & 46.3 & 47.2 & 49.4 & 48.0 \\
\hline Nonperforming/total loans (Forex) & 10.7 & 30.1 & 20.3 & 16.7 & 22.0 & 26.0 & 38.1 \\
\hline Nonperforming/total loans (Togrog) & 20.8 & 53.8 & 32.8 & 56.9 & 54.8 & 60.5 & 60.7 \\
\hline Forex current accounts/total current accounts & 59.5 & 44.2 & 57.4 & 49.2 & 60.2 & 64.8 & 58.8 \\
\hline Foreign currency deposits (US\$ million) & 59.9 & 41.7 & 60.6 & 44.2 & 42.8 & 50.2 & 51.4 \\
\hline \multicolumn{8}{|l|}{ Summary indicators (in percent) } \\
\hline FC deposits/total deposits & 35.3 & 33.5 & 41.0 & 35.9 & 42.1 & 45.9 & 44.3 \\
\hline FC demand deposits/total demand deposits & 20.0 & 19.7 & 31.2 & 37.9 & 45.4 & 41.9 & 45.7 \\
\hline FC time and savings deposits/total time and savings deposit & 16.7 & 25.8 & 21.0 & 19.8 & 22.4 & 23.2 & 25.8 \\
\hline
\end{tabular}

Sources: Bank of Mongolia; and Fund staff estimates. 
Table 43. Mongolia: External Debt and Debt Service in Convertible Currencies, 1994-98 1/

\begin{tabular}{|c|c|c|c|c|c|}
\hline & 1994 & 1995 & 1996 & 1997 & $19982 /$ \\
\hline & \multicolumn{5}{|c|}{ (In millions of U.S. dollars) } \\
\hline External debt $1 /$ & 473.7 & 503.8 & 542.2 & 604.8 & 753.1 \\
\hline Medium and long term & 418.5 & 456.8 & 498.6 & 557.3 & 704.8 \\
\hline $\mathrm{IMF}$ & 55.2 & 47.0 & 43.8 & 47.6 & 48.3 \\
\hline Short-term & 0.0 & 0.0 & 0.0 & 0.0 & 0.0 \\
\hline Debt service & 66.7 & 58.3 & 43.3 & 35.5 & 26.2 \\
\hline Amortization & 52.4 & 49.1 & 36.1 & 29.5 & 16.7 \\
\hline Interest & 14.3 & 9.2 & 7.2 & 6.0 & 9.5 \\
\hline Medium and long term & 65.6 & 47.6 & 32.8 & 34.5 & 24.7 \\
\hline Amortization & 52.4 & 39.4 & 26.1 & 28.7 & 15.5 \\
\hline Interest & 13.2 & 8.2 & 6.7 & 5.8 & 9.2 \\
\hline IMF & 1.1 & 10.8 & 10.5 & 1.1 & 1.5 \\
\hline Repurchase/Repayments & 0.0 & 9.7 & 10.0 & 0.9 & 1.3 \\
\hline Charges & 1.1 & 1.1 & 0.5 & 0.2 & 0.2 \\
\hline \multirow[t]{2}{*}{ Short term } & 0.0 & 0.0 & 0.0 & 0.0 & 0.0 \\
\hline & \multicolumn{5}{|c|}{ (In percent) } \\
\hline External debt/GDP & 68.7 & 52.8 & 54.0 & 63.3 & 73.4 \\
\hline Medium and long term & 60.7 & 47.9 & 49.6 & 58.4 & 68.7 \\
\hline $\mathrm{IMF}$ & 8.0 & 4.9 & 4.4 & 5.0 & 4.7 \\
\hline Short term & 0.0 & 0.0 & 0.0 & 0.0 & 0.0 \\
\hline External debt/exports goods and services & 116.4 & 95.4 & 113.2 & 93.3 & 139.4 \\
\hline Medium and long term & 102.9 & 86.5 & 104.1 & 85.9 & 130.5 \\
\hline $\mathbb{M M F}$ & 13.6 & 8.9 & 9.1 & 7.3 & 8.9 \\
\hline Short term & 0.0 & 0.0 & 0.0 & 0.0 & 0.0 \\
\hline Debt service ratio & 16.4 & 11.0 & 9.0 & 5.5 & 4.9 \\
\hline Amortization & 12.9 & 9.3 & 7.5 & 4.5 & 3.1 \\
\hline Interest & 3.5 & 1.7 & 1.5 & 0.9 & 1.8 \\
\hline Medium and long term & 16.1 & 9.0 & 6.8 & 5.3 & 4.6 \\
\hline Amortization & 12.9 & 7.5 & 5.4 & 4.4 & 2.9 \\
\hline Interest & 3.2 & 1.6 & 1.4 & 0.9 & 1.7 \\
\hline $\mathrm{IMF}$ & 0.3 & 2.0 & 2.2 & 0.2 & 0.3 \\
\hline Repurchase/repayments & 0.0 & 1.8 & 2.1 & 0.1 & 0.2 \\
\hline Charges & 0.3 & 0.2 & 0.1 & 0.0 & 0.0 \\
\hline Short term & 0.0 & 0.0 & 0.0 & 0.0 & 0.0 \\
\hline
\end{tabular}

Sources: Mongolian authorities; and Fund staff estimates.

1/ Data refers to actual payments.

2/ Debt stock includes debt in arrears. 
Table 44. Mongolia: Monthly Exchange Rates, January 1996-September 1999

(In togrogs per U.S. dollar, period average)

\begin{tabular}{|c|c|c|c|}
\hline & Official Rate 1/ & Interbank Rate 2/ & Street Rate \\
\hline \multicolumn{4}{|l|}{1996} \\
\hline January & 476.0 & 481.4 & 492.2 \\
\hline February & 481.9 & 489.3 & 501.5 \\
\hline March & 486.5 & 494.1 & 510.0 \\
\hline April & 491.7 & 500.9 & 508.0 \\
\hline May & 504.8 & 506.0 & 515.9 \\
\hline June & 524.9 & 528.8 & 549.4 \\
\hline July & 534.3 & 543.1 & 558.0 \\
\hline August & 544.2 & 561.0 & 569.0 \\
\hline September & 586.5 & 595.8 & 600.6 \\
\hline October & 606.2 & 611.5 & 614.3 \\
\hline November & 655.8 & 676.7 & 679.0 \\
\hline December & 692.4 & 693.9 & 692.8 \\
\hline \multicolumn{4}{|l|}{1997} \\
\hline January & 710.6 & 718.0 & $724.0^{\circ}$ \\
\hline February & 733.9 & 749.3 & 731.8 \\
\hline March & 819.2 & 844.4 & 807.4 \\
\hline April & 805.0 & 801.1 & 818.4 \\
\hline May & 787.1 & 786.9 & 785.6 \\
\hline June & 796.8 & 797.6 & 795.8 \\
\hline July & 799.1 & 800.1 & 800.2 \\
\hline August & 803.7 & 806.7 & 807.7 \\
\hline September & 803.8 & 806.6 & 807.8 \\
\hline October & 804.9 & 808.1 & 809.9 \\
\hline November & 808.1 & 810.3 & 811.9 \\
\hline December & 811.9 & 812.2 & 813.6 \\
\hline \multicolumn{4}{|l|}{1998} \\
\hline Jamuary & 815.2 & 816.9 & 821.2 \\
\hline February & 816.5 & 818.2 & 820.9 \\
\hline March & 816.7 & 817.6 & 821.1 \\
\hline April & 817.9 & 818.5 & 820.6 \\
\hline May & 821.9 & 823.9 & 831.1 \\
\hline June & 833.6 & 838.9 & 849.6 \\
\hline July & 839.3 & 842.4 & 852.1 \\
\hline August & 846.4 & 853.5 & 866.6 \\
\hline September & 852.0 & 856.0 & 863.6 \\
\hline October & 857.9 & 863.9 & 871.8 \\
\hline November & 872.5 & 880.5 & 889.1 \\
\hline December & 891.9 & 905.4 & 911.9 \\
\hline \multicolumn{4}{|l|}{1999} \\
\hline January & 916.5 & 929.7 & 944.1 \\
\hline February & 975.2 & 982.0 & 986.5 \\
\hline March & 1019.6 & 1049.2 & 1066.3 \\
\hline April & 1024.9 & 1017.5 & 1014.5 \\
\hline May & 1004.4 & 1001.3 & 1003.3 \\
\hline June & 1008.1 & 1008.9 & 1012.7 \\
\hline July & 1015.0 & 1017.1 & 1020.7 \\
\hline August & 1035.2 & 1042.4 & 1047.5 \\
\hline September & 1052.5 & 1055.6 & 1061.0 \\
\hline
\end{tabular}

Source: Bank of Mongolia.

1/ Midpoint rate announced by the Bank of Mongolia.

2/ Midpoint rate established in the interbank foreign exchange market. 
Table 45. Mongolia: Extemal Debt Stock by Creditor, 1996-98 1/

\begin{tabular}{|c|c|c|c|c|c|c|}
\hline & 1996 & 1997 & 1998 & 1996 & 1997 & 1998 \\
\hline & \multicolumn{3}{|c|}{ (In millions of U.S. dollars) } & \multicolumn{3}{|c|}{ (In percent of external debt) } \\
\hline External Debt & 542.2 & 604.8 & 753.1 & 100.0 & 100.0 & 100.0 \\
\hline Medium and long-term official & 443.4 & 518.0 & 647.0 & 81.8 & 85.6 & 85.9 \\
\hline Multilateral (excluding IMF) & 205.0 & 288.0 & 350.8 & 37.8 & 47.6 & 46.6 \\
\hline Asian Development Bank & 137.3 & 193.3 & 234.7 & 25.3 & 32.0 & 31.2 \\
\hline World Bank & 67.7 & 94.4 & 115.5 & 12.5 & 15.6 & 15.3 \\
\hline Bilateral official & 238.4 & 229.9 & 296.3 & 44.0 & 38.0 & 39.3 \\
\hline Paris Club & 225.2 & 212.3 & 275.4 & 41.5 & 35.1 & 36.6 \\
\hline Japan & 112.1 & 112.3 & 171.6 & 20.7 & 18.6 & 22.8 \\
\hline Russia & 73.3 & 51.9 & 50.2 & 13.5 & 8.6 & 6.7 \\
\hline Germany & 30.9 & 31.7 & 36.0 & 5.7 & 5.2 & 4.8 \\
\hline Finland & 6.9 & 8.2 & 6.2 & 1.3 & 1.4 & 0.8 \\
\hline Norway & 2.0 & 8.2 & 11.4 & 0.4 & 1.4 & 1.5 \\
\hline Non-Paris Club & 13.2 & 17.6 & 20.9 & 2.4 & 2.9 & 2.8 \\
\hline China & 5.9 & 13.0 & 14.3 & 1.1 & 2.2 & 1.9 \\
\hline Korea & 4.8 & 2.4 & 2.9 & 0.9 & 0.4 & 0.4 \\
\hline India & 1.6 & 1.5 & 1.3 & 0.3 & 0.2 & 0.2 \\
\hline Hungary & 0.9 & 0.0 & 0.0 & 0.2 & 0.0 & 0.0 \\
\hline Kuwait & 0.0 & 0.7 & 2.4 & 0.0 & 0.1 & 0.3 \\
\hline IMF & 43.6 & 47.6 & 48.3 & 8.0 & 7.9 & 6.4 \\
\hline Commercial & 55.2 & 39.3 & 57.8 & 10.2 & 6.5 & 7.7 \\
\hline Short-term & 0.0 & 0.0 & 0.0 & 0.0 & 0.0 & 0.0 \\
\hline
\end{tabular}

Sources: Bank of Mongolia, and the Ministry of Finance; and Fund staff estimates.

1/ Excludes unresolved transferable ruble claims. 
Table 46. Mongolia: Official Grants, 1994-99 1/

(In millions of U.S. dollars)

\begin{tabular}{lrrrrrr}
\hline & 1994 & 1995 & 1996 & 1997 & 1998 & $\begin{array}{r}1999 \\
\text { Jan.-Sep. }\end{array}$ \\
\hline Japan & & & & & & \\
United States & 44.8 & 47.0 & 45.2 & 34.0 & 24.0 & 31.3 \\
Denmark & 11.5 & 2.7 & 3.7 & 7.0 & 10.1 & 3.1 \\
Germany & 8.2 & 0.7 & 0.3 & 0.8 & 0.1 & 0.1 \\
Korea & 1.4 & 13.5 & 0.8 & 0.6 & 1.1 & 0.6 \\
China & 0.5 & 0.1 & 0.0 & 0.0 & 0.5 & 0.3 \\
Italy & 0.0 & 0.0 & 0.0 & 0.0 & 0.1 & 0.6 \\
Netherlands & 1.3 & 0.0 & 0.0 & 0.0 & 0.0 & 0.0 \\
France & 0.0 & 3.8 & 0.9 & 0.7 & 0.0 & 0.0 \\
United Kingdom & 0.0 & 0.0 & 0.0 & 0.0 & 2.4 & 3.5 \\
Other countries & 0.8 & 1.0 & 0.0 & 0.0 & 0.2 & 0.0 \\
United Nations & 0.1 & 1.5 & 0.3 & 2.4 & 6.4 & 3.4 \\
European Union & & & & & & \\
$\quad$ Total & 0.9 & 0.0 & 2.0 & 5.8 & 0.7 & 0.8 \\
& 0.0 & 0.6 & 3.0 & 3.5 & 0.0 & 0.1 \\
\hline
\end{tabular}

Sources: Mongolian authorities; and Fund staff estimates.

1/ Excludes technical assistance. 


\section{Mongolia-Trade Regime}

\section{Trade regime}

1. Mongolia maintains a liberal trade regime with a low uniform level of import tariffs of 5 percent, a limited number of export taxes and no significant non-tariff trade restrictions (Table 1). It has a minimum level 1 of the trade restrictiveness rating computed by the IMF. The import tariffs levels are among the lowest in the Asian region. Between May 1997 and July 1999 there were no import tariffs (0 rate).

2. Prior to 1991, Mongolia maintained a system of the multiple custom tariffs. A number of products were subject to export and import prohibitions. In addition, a regime of (non-automatic) licensing applied to many products, including meat, grain, fodder, refined spirits, and alcoholic beverages. The export of raw cashmere was prohibited. Mongolia also maintained substantial export subsidies for sectors such as mining, metal processing, machinery, and infrastructure.

3. Mongolia applied for membership to the World Trade Organization (WTO) in 1991. As part of the accession procedures, the trade system was gradually liberalized. Mongolia joined the WTO in January 1997. Under the rules of accession, Mongolia agreed to bind its entire tariff schedule at a ceiling rate of 20 percent with some exceptions and specific tariff concessions. Most of the ceilings were higher than the rate prevailing at that time of 15 percent. The prohibition on exports of raw cashmere was replaced by an export tax, which Mongolia agreed to phase out within 10 years of the date of accession. Shortly after the accession to the WTO all import tariffs were abolished on May 1, 1997. On July 1, 1999 a uniform import tariff rate of 5 percent was introduced with exceptions for a few goods.

4. Mongolia receives benefits under the Generalized System of Preferences as administered by Australia, Japan, New Zealand, Norway, Switzerland, the European Union countries, Bulgaria, Czech Republic, Russia, and Slovakia.

\section{Procedures}

5. Export and import procedures are regulated by the Customs Law. There are 33 permanent and temporary customs duty ports. As part of its membership of the WTO, Mongolia is committed to adhere to the WTO custom valuation agreement as of January 2000. The main changes required under this agreement relate to standardization of the custom forms and the adoption of the unified transaction method for valuation of imported goods.

\footnotetext{
${ }^{1}$ Sources: IMF Trade Policy Information Database (TPID). For more information on the construction of the trade restrictiveness rating, see "Trade Liberalization in IMF-supported Programs (IMF, 1998).
} 
6. For clearance of goods, exporters and importers are required to fill out a declaration form and pay the prescribed duties/taxes. They may also be required to submit relevant documents, including trade contracts, invoices, transport documents, packing lists, certificates of origin, verification of prior payments of taxes, bank securities, and import licenses and permits (where applicable).

7. Gold and other precious metals may be exported through the Bank of Mongolia (BOM), authorized commercial banks, or by holders of mining licenses themselves. In all cases, the amount and content of exports is subject to registration at the BOM. In addition, the exports need to be certified by the Precious Metals Grading Bureau of the Ministry of Finance. Exporters are charged for the costs of the laboratory tests. 
Table 1. Mongolia-Summary of Trade Taxes and Restrictions in effect as of November 1999

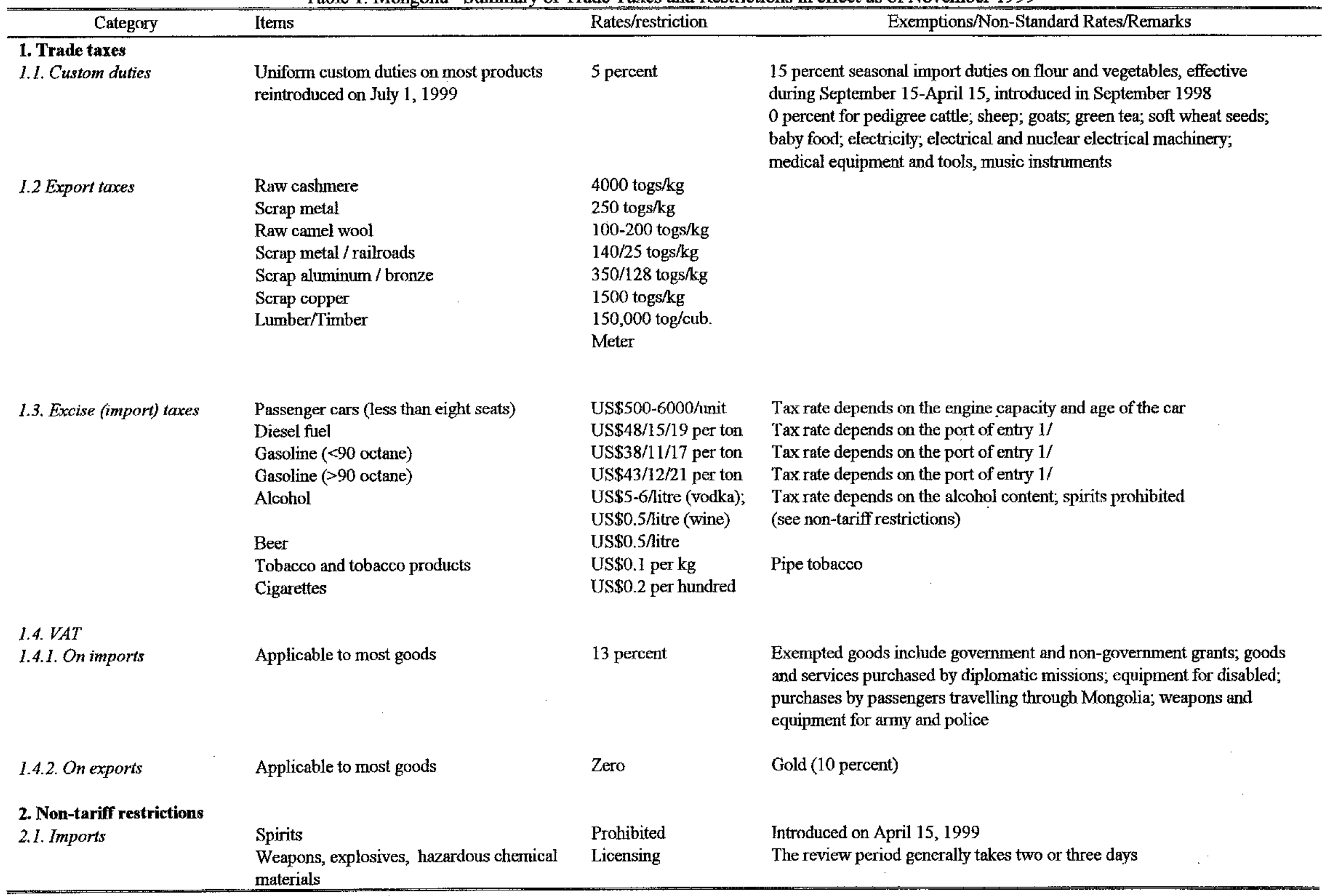

1/ Central, Western, and North-western, respectively. 